\title{
HECKMAN SELECTION-T MODEL: PARAMETER ESTIMATION VIA THE EM-ALGORITHM
}

\author{
Victor H. Lachos Davila \\ Department of Statistics \\ University of Connecticut \\ Storrs, CT 06269 \\ hlachoseuconn. edu
}

\author{
Marcos O. Prates \\ Departamento de Estadística \\ Universidad Federal de Minas Gerais \\ Minas Gerai, MG, Brazil \\ marcosopdest.ufmg.br
}

\author{
Dipak K. Dey \\ Department of Statistics \\ University of Connecticut \\ Storrs, CT 06250 \\ dipak. dey@uconn.edu
}

June 16,2020

\begin{abstract}
Heckman selection model is perhaps the most popular econometric model in the analysis of data with sample selection. The analyses of this model are based on the normality assumption for the error terms, however, in some applications, the distribution of the error term departs significantly from normality, for instance, in the presence of heavy tails and/or atypical observation. In this paper, we explore the Heckman selection-t model where the random errors follow a bivariate Student's-t distribution. We develop an analytically tractable and efficient EM-type algorithm for iteratively computing maximum likelihood estimates of the parameters, with standard errors as a by-product. The algorithm has closed-form expressions at the E-step, that rely on formulas for the mean and variance of the truncated Student's-t distributions. Simulations studies show the vulnerability of the Heckman selection-normal model, as well as the robustness aspects of the Heckman selection-t model. Two real examples are analyzed, illustrating the usefulness of the proposed methods. The proposed algorithms and methods are implemented in the new R package HeckmanEM.
\end{abstract}

Keywords EM-type algorithms $\cdot$ Heckman selection model $\cdot$ Multivariate Student's- $t \cdot$ Robustness.

\section{Introduction}

Sample selection (SL) often occurs many fields, like economics, biostatistics, finance, medical surveys, sociology and political science, to name a few. For example, in a sample of individual consumers with expenditures below or above some threshold, where the unobserved random variable (decision to spend) is related to the spending amount. As expenditure is not independent of the decision to spend, this sample may represent only a subset of the full population, and thus selection bias arises. In a classic example, Heckman (1979) proposed the SL model, aiming to estimate the wage offer function of women. Because housewives wages are not observed, the sample collected is subject to the self-selection problem. SL is a special case of a more general concept known in the econometrics literature as limited dependent variables or variables observed over a limited range of their support, however, censoring (Massuia et al. 2015) is much simpler than SL.

The classical Heckman SL model was introduced by Heckman (1974) when he proposed a parametric approach to the parameter estimation under the assumption of bivariate normality (SLn). However, it is well-known that several phenomena are not always in agreement with this assumption, yielding residuals with a distribution with heavy tails or skewness. These characteristics can be circumvented by data transformations, as proposed by Lee (1983), which can render approximate normality with reasonable empirical results. However, some possible drawbacks of these methods are: (i) transformations provide reduced information on the underlying data generation scheme; (ii) component wise transformations may not guarantee joint normality; (iii) parameters may lose interpretability in a transformed scale and (iv) transformations may not be universal and usually vary with the data set. Hence, from a practical perspective, there is a need to seek an appropriate theoretical model that avoids data transformations. 
There are two-ways of estimating the SLn model, via maximum likelihood (ML) and using a two-step procedure (Heckman, 1979). A drawback of the ML estimation is less robust than the two-step procedure and is sometimes difficult to get it to converge (Wooldridge, 2010). However, ML estimation will be more efficient if the random error really are jointly normally distributed. In this context, many robust methods have been proposed over the years to broaden the applicability of the SLn model to situations where the Gaussian error term assumption may be inadequate. For instance, the semiparametric SL model proposed by Ahn and Powell (1993) and the nonparametric SL model proposed by Das et al. (2003). From a Bayesian perspective, Kai (1998) proposed a Bayesian inference procedure for the SL model using data augmentation. More recently, Kim et al. (2019) proposed a flexible nonparametric SL model using Bernstein Polynomial.

In the context of parametric models, Marchenko and Genton (2012) introduced the Heckman selection-t model (SLt) model that extends the conventional SLn model by Heckman (1979) to have a bivariate Student's t error distribution. This model provides a greater flexibility for modeling heavier-tailed data than the SLn model by introducing only one extra parameter, the degrees of freedom, controlling the tails of the distribution. The authors considered ML estimation of the parameters via Newton-Raphson procedures available in statistical packages such as R and stata. They demonstrated the robustness aspects of the SLt model against outliers through extensive simulations. More recently, Zhao et al. (2020) have proposed EM-type algorithms for ML estimation of the SLn model, which have the advantages of easy implementation and numerical stability. Moreover, ML estimation via the EM algorithm yield better estimators than the 2-step procedure.

Motivated by Zhao et al. (2020), in this paper we propose a novel, simple and efficient EM-type algorithm for iteratively computing ML estimates of the parameters in the SLt model. We show that the E-step reduces to computing the first two moments of a truncated multivariate Student's t distribution. The general formulas for these moments were derived efficiently by Galarza et al. (2020), for which we use the MomTrunc package in R. The likelihood function is easily computed as a byproduct of the E-step and is used for monitoring convergence and for model selection (AIC and BIC). Furthermore, we consider a general information-based method for obtaining the asymptotic covariance matrix of the ML estimate. The method proposed in this paper is implemented in the R package HeckmanEM, which is available for download from Github (https://github.com/marcosop/HeckmanEM).

The remainder of the paper is organized as follows. In Section 2, we briefly discuss some preliminary results related to the multivariate Student's- $t$ distribution and its truncated version. Some of its key properties are also discussed. In Section 3 , we present the SLn model proposed by Heckman (1979) and the related EM algorithm for ML estimation. In Section 4, we present the robust SLt model, including the EM-type algorithm for ML estimation, and derive the empirical information matrix analytically to obtain the standard errors. In Sections 5 and 6 , numerical examples using both simulated and real data are given to illustrate the performance of the proposed method. Finally, some concluding remarks are presented in Section 7

\section{Background}

In this section, we present some useful results associated with the $p$-variate Student's- $t$ distribution and its truncated version, the $p$-variate truncated Student's- $t$ distribution. We begin our exposition by defining the notation and presenting some basic concepts which are used throughout the development of our methodology. As is usual in probability theory and its applications, we denote a random variable by an upper-case letter and its realization by the corresponding lower case and use boldface letters for vectors and matrices. Let $\mathbf{I}_{p}$ represent a $p \times p$ identity matrix, $\mathbf{A}^{\top}$ be the transpose of $\mathbf{A}$. For multiple integrals, we use the shorthand notation

$$
\int_{\mathbf{a}}^{\mathbf{b}} f(\mathbf{x}) d \mathbf{x}=\int_{a_{1}}^{b_{1}} \ldots \int_{a_{p}}^{b_{p}} f\left(x_{1}, \ldots, x_{p}\right) \mathrm{d} x_{p} \ldots \mathrm{d} x_{1} .
$$

where $\mathbf{a}=\left(a_{1}, \ldots, a_{p}\right)^{\top}$ and $\mathbf{b}=\left(b_{1}, \ldots, b_{p}\right)^{\top}$. If the Borel set in $\mathbb{R}^{p}$ has the form

$$
\mathbb{A}=\left\{\left(x_{1}, \ldots, x_{p}\right) \in \mathbb{R}^{p}: a_{1} \leq x_{1} \leq b_{1}, \ldots, a_{p} \leq x_{p} \leq b_{p}\right\}=\left\{\mathbf{x} \in \mathbb{R}^{p}: \mathbf{a} \leq \mathbf{x} \leq \mathbf{b}\right\} .
$$

we use the notation $\{\mathbf{Y} \in \mathbb{A}\}=\{\mathbf{a} \leq \mathbf{Y} \leq \mathbf{b}\}$.

\subsection{The multivariate Student's- $t$ distribution}

A random variable $\mathbf{X}$ having a $p$-variate Student's- $t$ distribution with location vector $\boldsymbol{\mu}$, positive-definite scalecovariance matrix $\boldsymbol{\Sigma}$ and degrees of freedom $\nu$, denoted by $\mathbf{X} \sim t_{p}(\boldsymbol{\mu}, \boldsymbol{\Sigma}, \nu)$, has the pdf:

$$
t_{p}(\mathbf{x} \mid \boldsymbol{\mu}, \boldsymbol{\Sigma}, \nu)=\frac{\Gamma\left(\frac{p+\nu}{2}\right)}{\Gamma\left(\frac{\nu}{2}\right) \pi^{p / 2}} \nu^{-p / 2}|\boldsymbol{\Sigma}|^{-1 / 2}\left(1+\frac{\delta(\mathbf{x})}{\nu}\right)^{-(p+\nu) / 2}
$$


where $\Gamma(\cdot)$ is the standard gamma function and $\delta(\mathbf{x})=(\mathbf{x}-\boldsymbol{\mu})^{\top} \boldsymbol{\Sigma}^{-1}(\mathbf{x}-\boldsymbol{\mu})$ is the squared Mahalanobis distance. Let $T_{p}(\mathbf{a}, \mathbf{b} ; \boldsymbol{\mu}, \boldsymbol{\Sigma}, \nu)$ represent

$$
T_{p}(\mathbf{a}, \mathbf{b} ; \boldsymbol{\mu}, \boldsymbol{\Sigma}, \nu)=\int_{\mathbf{a}}^{\mathbf{b}} t_{p}(\mathbf{x} \mid \boldsymbol{\mu}, \boldsymbol{\Sigma}, \boldsymbol{\nu}) \mathrm{d} \mathbf{x}
$$

where $\mathbf{a}=\left(a_{1}, \ldots, a_{p}\right)^{\top}$ and $\mathbf{b}=\left(b_{1}, \ldots, b_{p}\right)^{\top}$. When $\mathbf{a}=-\infty$ we will write simply $T_{p}(\mathbf{b} ; \boldsymbol{\mu}, \boldsymbol{\Sigma}, \nu)$ and when $p=1$ we will omit the sub-index $p$.

It is known that as $\nu \rightarrow \infty, \mathbf{X}$ converges in distribution to a multivariate normal with mean $\boldsymbol{\mu}$ and variance-covariance matrix $\boldsymbol{\Sigma}$, denoted by $N_{p}(\boldsymbol{\mu}, \boldsymbol{\Sigma})$. An important property of the random vector $\mathbf{X}$ is that it can be written as a scale mixture of the MVN random vector coupled with a positive random variable, i.e.,

$$
\mathbf{X}=\boldsymbol{\mu}+U^{-1 / 2} \mathbf{Z}
$$

where $\mathbf{Z} \sim N_{p}(\mathbf{0}, \boldsymbol{\Sigma})$, and is independent of $U \sim \operatorname{Gamma}(\nu / 2, \nu / 2)$, where $\operatorname{Gamma}(a, b)$ denotes a gamma distribution with mean $a / b$.

The following properties of the $p$-variate Student's- $t$ distribution are useful for our theoretical developments. We start with the marginal-conditional decomposition of a $p$-variate Student's- $t$ random vector. The proof of the following propositions can be found in Arellano-Valle and Bolfarine (1995).

Proposition 1 Let $\mathbf{X} \sim t_{p}(\boldsymbol{\mu}, \boldsymbol{\Sigma}, \nu)$ partitioned as $\mathbf{X}^{\top}=\left(\mathbf{X}_{1}^{\top}, \mathbf{X}_{2}^{\top}\right)^{\top}$ with $\operatorname{dim}\left(\mathbf{X}_{1}\right)=p_{1}, \operatorname{dim}\left(\mathbf{Y}_{2}\right)=p_{2}$, where $p_{1}+p_{2}=p$. Let $\boldsymbol{\mu}=\left(\boldsymbol{\mu}_{1}^{\top}, \boldsymbol{\mu}_{2}^{\top}\right)^{\top}$ and $\boldsymbol{\Sigma}=\left[\begin{array}{ll}\boldsymbol{\Sigma}_{11} & \boldsymbol{\Sigma}_{12} \\ \boldsymbol{\Sigma}_{21} & \boldsymbol{\Sigma}_{22}\end{array}\right]$ be the corresponding partitions of $\boldsymbol{\mu}$ and $\boldsymbol{\Sigma}$. Then, we have

(i) $\mathbf{X}_{1} \sim t_{p_{1}}\left(\boldsymbol{\mu}_{1}, \boldsymbol{\Sigma}_{11}, \nu\right)$; and

(ii) The conditional distribution of $\mathbf{X}_{2} \mid\left(\mathbf{X}_{1}=\mathbf{x}_{1}\right)$ is given by

$$
\mathbf{X}_{2} \mid\left(\mathbf{X}_{1}=\mathbf{x}_{1}\right) \sim t_{p_{2}}\left(\mathbf{y}_{2} \mid \boldsymbol{\mu}_{2.1}, \widetilde{\boldsymbol{\Sigma}}_{22.1}, \nu+p_{1}\right)
$$

where $\boldsymbol{\mu}_{2.1}=\boldsymbol{\mu}_{2}+\boldsymbol{\Sigma}_{21} \boldsymbol{\Sigma}_{11}^{-1}\left(\mathbf{x}_{1}-\boldsymbol{\mu}_{1}\right)$ and $\widetilde{\boldsymbol{\Sigma}}_{22.1}=\left(\frac{\nu+\delta_{1}}{\nu+p_{1}}\right) \boldsymbol{\Sigma}_{22.1}$ with $\delta_{1}=\left(\mathbf{x}_{1}-\boldsymbol{\mu}_{1}\right)^{\top} \boldsymbol{\Sigma}_{11}^{-1}\left(\mathbf{x}_{1}-\boldsymbol{\mu}_{1}\right)$ and $\boldsymbol{\Sigma}_{22.1}=\boldsymbol{\Sigma}_{22}-\boldsymbol{\Sigma}_{21} \boldsymbol{\Sigma}_{11}^{-1} \boldsymbol{\Sigma}_{12}$

Proposition 2 Let $\mathbf{X} \sim t_{p}(\boldsymbol{\mu}, \boldsymbol{\Sigma}, \nu)$. Then for any fixed vector $\mathbf{b} \in \mathbb{R}^{m}$ and matrix $\mathbf{A} \in \mathbb{R}^{m \times p}$ of full rank we get

$$
\mathbf{V}=\mathbf{b}+\mathbf{A} \mathbf{X} \sim t_{m}\left(\mathbf{b}+\mathbf{A} \boldsymbol{\mu}, \mathbf{A} \boldsymbol{\Sigma} \mathbf{A}^{\top}, \nu\right)
$$

\subsection{The multivariate truncated Student's- $t$ distribution}

A $p$-dimensional random vector $\mathbf{Y}$ is said to follow a doubly truncated Student's- $t$ distribution with location vector $\boldsymbol{\mu}$, scale-covariance matrix $\boldsymbol{\Sigma}$ and degrees of freedom $\nu$ over the truncation region $\mathbb{A}$ defined in $(1)$, denoted by $\mathbf{Y} \sim T t_{p}(\boldsymbol{\mu}, \boldsymbol{\Sigma}, \nu ; \mathbb{A})$, if it has the pdf:

$$
T t_{p}(\mathbf{y} \mid \boldsymbol{\mu}, \boldsymbol{\Sigma}, \nu ; \mathbb{A})=\frac{t_{p}(\mathbf{y} \mid \boldsymbol{\mu}, \boldsymbol{\Sigma}, \nu)}{T_{p}(\mathbf{a}, \mathbf{b} ; \boldsymbol{\mu}, \boldsymbol{\Sigma}, \nu)}, \mathbf{a} \leq \mathbf{y} \leq \mathbf{b}
$$

The cdf of $\mathbf{Y}$ evaluated at the region $\mathbf{a} \leq \mathbf{y} \leq \mathbf{b}$ is

$$
T T_{p}(\mathbf{y} \mid \boldsymbol{\mu}, \boldsymbol{\Sigma}, \nu ; \mathbb{A})=\frac{1}{T_{p}(\mathbf{a}, \mathbf{b} ; \boldsymbol{\mu}, \boldsymbol{\Sigma}, \nu)} \int_{\mathbf{a}}^{\mathbf{y}} t_{p}(\mathbf{x} \mid \boldsymbol{\mu}, \boldsymbol{\Sigma}, \nu) d \mathbf{x}=\frac{T_{p}(\mathbf{a}, \mathbf{y} ; \boldsymbol{\mu}, \mathbf{\Sigma}, \nu)}{T_{p}(\mathbf{a}, \mathbf{b} ; \boldsymbol{\mu}, \mathbf{\Sigma}, \nu)}
$$

The following propositions are related to the marginal and conditional moments of the first two moments of the TMVT distributions under a double truncation. The proof is similar to those given in Matos et al. (2013). In what follows, we shall use the notation $\mathbf{Y}^{(0)}=1, \mathbf{Y}^{(1)}=\mathbf{Y}, \mathbf{Y}^{(2)}=\mathbf{Y} \mathbf{Y}^{\top}$, and $\mathbf{W} \sim T t_{p}(\boldsymbol{\mu}, \boldsymbol{\Sigma}, \nu ;(\mathbf{a}, \mathbf{b}))$ stands for a $p$-variate doubly truncated Student's- $t$ distribution on $(\mathbf{a}, \mathbf{b}) \in \mathbb{R}^{p}$.

Proposition 3 If $\mathbf{Y} \sim T t_{p}(\boldsymbol{\mu}, \boldsymbol{\Sigma}, \nu ;(\mathbf{a}, \mathbf{b}))$ then it follows that

$$
\mathbb{E}\left[\left(\frac{\nu+p}{\nu+\delta(\mathbf{Y})}\right)^{r} \mathbf{Y}^{(k)}\right]=c_{p}(\nu, r) \frac{T_{p}\left(\mathbf{a}, \mathbf{b} ; \boldsymbol{\mu}, \boldsymbol{\Sigma}^{*}, \nu+2 r\right)}{T_{p}(\mathbf{a}, \mathbf{b} ; \boldsymbol{\mu}, \boldsymbol{\Sigma}, \nu)} \mathbb{E}\left[\mathbf{W}^{(k)}\right],
$$


where

$$
\begin{aligned}
& c_{p}(\nu, r)=\left(\frac{\nu+p}{\nu}\right)^{r} \frac{\Gamma\left(\frac{p+\nu}{2}\right) \Gamma\left(\frac{\nu+2 r}{2}\right)}{\Gamma\left(\frac{\nu}{2}\right) \Gamma\left(\frac{p+\nu+2 r}{2}\right)}, \\
& \mathbf{\Sigma}^{*}=\nu \boldsymbol{\Sigma} /(\nu+2 r) \text { and } \nu+2 r>0, \text { with } \mathbf{W} \sim T t_{p}\left(\boldsymbol{\mu}, \boldsymbol{\Sigma}^{*}, \nu+2 r ;(\mathbf{a}, \mathbf{b})\right) .
\end{aligned}
$$

Notice that Proposition 3 depends on formulas for $\mathbb{E}[\mathbf{W}]$ and $\mathbb{E}\left[\mathbf{W} \mathbf{W}^{\top}\right]$, where $\mathbf{W} \sim T t_{p}(\boldsymbol{\mu}, \boldsymbol{\Sigma}, \nu ;(\mathbf{a}, \mathbf{b}))$. Having established the formula on the $k$-order moment of $\mathbf{Y}$, we provide an explicit formula for the conditional moments with respect to a two-component partition of $\mathbf{Y}$.

Proposition 4 Let $\mathbf{Y} \sim T t_{p}(\boldsymbol{\mu}, \boldsymbol{\Sigma}, \nu ;(\mathbf{a}, \mathbf{b}))$. Consider the partition $\mathbf{Y}^{\top}=\left(\mathbf{Y}_{1}^{\top}, \mathbf{Y}_{2}^{\top}\right)$ with $\operatorname{dim}\left(\mathbf{Y}_{1}\right)=p_{1}$, $\operatorname{dim}\left(\mathbf{Y}_{2}\right)=p_{2}, p_{1}+p_{2}=p$, and the corresponding partitions of $\mathbf{a}, \mathbf{b}, \boldsymbol{\mu}$, and $\boldsymbol{\Sigma}$. Then,

$$
\mathbb{E}\left[\left(\frac{\nu+p}{\nu+\delta(\mathbf{Y})}\right)^{r} \mathbf{Y}_{2}^{(k)} \mid \mathbf{Y}_{1}\right]=\frac{d_{p}\left(p_{1}, \nu, r\right)}{\left(\nu+\delta\left(\mathbf{Y}_{1}\right)\right)^{r}} \frac{T_{p_{2}}\left(\mathbf{a}_{2}, \mathbf{b}_{2} ; \boldsymbol{\mu}_{2.1}, \widetilde{\boldsymbol{\Sigma}}_{22.1}^{*}, \nu+p_{1}+2 r\right)}{T_{p_{2}}\left(\mathbf{a}_{2}, \mathbf{b}_{2} ; \boldsymbol{\mu}_{2.1}, \widetilde{\boldsymbol{\Sigma}}_{22.1}, \nu+p_{1}\right)} \mathbb{E}\left[\mathbf{W}_{2}^{(k)}\right],
$$

for $\nu+p_{1}+2 r>0$, with $\delta\left(\mathbf{Y}_{1}\right)=\delta\left(\mathbf{Y}_{1} ; \boldsymbol{\mu}_{1}, \boldsymbol{\Sigma}_{11}\right)$,

$$
\widetilde{\mathbf{\Sigma}}_{22.1}^{*}=\left(\frac{\nu+\delta_{1}}{\nu+2 r+p_{1}}\right) \boldsymbol{\Sigma}_{22.1}, \quad \text { and } \quad d_{p}\left(p_{1}, \nu, r\right)=(\nu+p)^{r} \frac{\Gamma\left(\frac{p+\nu}{2}\right) \Gamma\left(\frac{p_{1}+\nu+2 r}{2}\right)}{\Gamma\left(\frac{p_{1}+\nu}{2}\right) \Gamma\left(\frac{p+\nu+2 r}{2}\right)},
$$

where $\boldsymbol{\Sigma}_{22.1}$ is defined as in proposition 1$]$ Moreover, $\mathbf{W}_{2} \sim T t_{p_{2}}\left(\boldsymbol{\mu}_{2.1}, \widetilde{\mathbf{\Sigma}}_{22.1}^{*}, \nu+p_{1}+2 r ;\left[\mathbf{a}_{2}, \mathbf{b}_{2}\right]\right)$.

Observe that propositions 3 and 4 depend on formulas for $\mathrm{E}[\mathbf{W}]$ and $\mathrm{E}\left[\mathbf{W} \mathbf{W}^{\top}\right]$, where $\mathbf{W} \sim T t_{p}(\boldsymbol{\mu}, \boldsymbol{\Sigma}, \nu ; \mathbb{A})$. The general formulas for these moments were derived efficiently by Galarza et al. (2020), for which we use the MomTrunc package in $R$.

\section{Review of the Heckman selection normal model}

Sample selection or missing data is common in applied research. The SL model consists of a linear equation for the outcome, and a Probit equation for the sample selection mechanism. The outcome equation is

$$
Y_{1 i}=\mathbf{x}_{i}^{\top} \boldsymbol{\beta}+\epsilon_{1 i},
$$

and the sample selection mechanism is characterized by the following latent linear equation:

$$
Y_{2 i}=\mathbf{w}_{i}^{\top} \gamma+\epsilon_{2 i},
$$

for $i=1, \ldots, n$. The vectors $\boldsymbol{\beta} \in \mathbb{R}^{p}$ and $\boldsymbol{\gamma} \in \mathbb{R}^{q}$ are unknown regression parameters. $\mathbf{x}_{i}^{\top}=\left(x_{i 1}, \ldots, x_{i p}\right)$ and $\mathbf{w}_{i}^{\top}=\left(w_{i 1}, \ldots, w_{i q}\right)$ are known characteristics. The covariates in $\mathbf{x}_{i}$ and $\mathbf{w}_{i}$ may overlap with each other, and the exclusion restriction holds when at least one of the elements of $\mathbf{w}_{i}$ are not in $\mathbf{x}_{i}$. The indicator for sample selection is $C_{i}=I\left(Y_{2 i}>0\right)$. Let $V_{1 i}$ be the observed outcome, we observe the outcome $V_{1 i}$ if and only if $C_{i}>0$, i.e., $Y_{1 i}=V_{1 i}$ if $C_{i}=1$, and $Y_{1 i}=N A$ if $C_{i}=0$, where $N A$ indicates missing data.

Heckman (1979) assumes independent $(\stackrel{\text { ind. }}{\sim})$ bivariate normal distribution for the error terms (SLn), as follows:

$$
\left[\begin{array}{c}
\epsilon_{1 i} \\
\epsilon_{2 i}
\end{array}\right] \stackrel{\text { ind. }}{\sim} N_{2}\left(\left[\begin{array}{l}
0 \\
0
\end{array}\right], \boldsymbol{\Sigma}=\left[\begin{array}{cc}
\sigma^{2} & \rho \sigma \\
\rho \sigma & 1
\end{array}\right]\right)
$$

where the second diagonal element of $\boldsymbol{\Sigma}$ is fixed at 1 in order to achieve full identifiability. The SLn model (3)-(5) is known as "Type 2 tobit model" in the econometrics literature and is sometimes also referred to as the Heckman model. Absence of selection effect $(\rho=0)$ implies that the outcomes are missing at random, and the observed outcomes are representative for inference of the population given the observed covariates.

Under the bivariate normal assumption, the mean equation for the outcomes if the selected samples is

$$
\mathbb{E}\left[Y_{1 i} \mid C_{i}=1, \mathbf{x}_{i}, \mathbf{w}_{i}\right]=\mathbf{x}_{i}^{\top} \boldsymbol{\beta}+\rho \sigma \lambda\left(\mathbf{w}_{i}^{\top} \boldsymbol{\gamma}\right),
$$


where $\lambda(a)=\frac{\phi(a)}{\Phi(a)}$ is the inverse Mills ratio, $\phi($.$) and \Phi($.$) denote the pdf and cdf of the standard normal distribution,$ respectively. Therefore, the SLn problem can be treated as a model misspecification problem, because the mean equation for the outcomes of the selected samples is a linear function $\mathbf{x}_{i}^{\top} \boldsymbol{\beta}$ with a nonlinear correction term $\rho \sigma \lambda\left(\mathbf{w}_{i}^{\top} \boldsymbol{\gamma}\right)$. Based on (6), Heckman (1979) proposed a two-step procedure by first fitting a Probit model of $C_{i}$ on $\mathbf{w}_{i}$ to obtain $\hat{\boldsymbol{\gamma}}$. At the second stage, $\boldsymbol{\beta}$ and $\rho^{*}=\sigma \rho$ are estimated by least squares regression of $Y_{1 i}$ (the observed counterpart) on $\mathbf{x}_{i}$ and $\hat{\lambda}=\frac{\phi\left(\mathbf{w}_{i}^{\top} \hat{\gamma}\right)}{\Phi\left(\mathbf{w}_{i}^{\top} \hat{\gamma}\right)}$. The consistent estimators of $\rho$ and $\sigma$ can then be obtained from $\hat{\rho}^{*}$, least square residual variance, and average-predicted probabilities from the probit model. The two-step procedure is less efficient than the ML estimation, but it is robust to the deviation of the joint normality of the error terms. The ML estimates of the SLn model can be calculated by Newton-Raphson iteration or the EM algorithm as discussed by Zhao et al. (2020). In the next subsection, we propose a slight modification to the EM-type algorithms proposed by Zhao et al. (2020), wherein all the parameters are updated (M-step) by considering the outcome $\left(Y_{1 i}\right)$ and sample selection $\left(Y_{2 i}\right)$ as missing data (Vaida and Liu, 2009; Matos et al. 2013).

Ignoring censoring for the moment, suppose that we have observations on $n$ independent individuals

$$
\mathbf{Y}_{1}, \ldots, \mathbf{Y}_{n} \stackrel{\text { ind. }}{\sim} N_{2}\left(\boldsymbol{\mu}_{i}, \boldsymbol{\Sigma}\right)
$$

where for each $i \in\{1, \ldots, n\}, \mathbf{Y}_{i}=\left(Y_{1 i}, Y_{2 i}\right)^{\top}$ is the vector of independent responses for sample unit $i$,

$$
\boldsymbol{\mu}_{i}=\mathbf{X}_{i c} \boldsymbol{\beta}_{c}, \text { with } \mathbf{X}_{i c}=\left[\begin{array}{cc}
\mathbf{x}_{i}^{\top} & 0 \\
0 & \mathbf{w}_{i}^{\top}
\end{array}\right], \quad \boldsymbol{\beta}_{c}=\left[\begin{array}{l}
\boldsymbol{\beta} \\
\boldsymbol{\gamma}
\end{array}\right]
$$

and the dispersion matrix $\boldsymbol{\Sigma}$ depends on an unknown parameter vector $(\sigma, \rho)$. We consider the approach proposed by Vaida and Liu (2009) and Matos et al. (2013) to represent the model belong to the structure of a censored linear model. Thus, the observed data for the $i$ th subject is given by $\left(\mathbf{V}_{i}, C_{i}\right)$, where $\mathbf{V}_{i}$ represents the vector of censored readings and $C_{i}=I_{\left\{Y_{2 i}>0\right\}}$ is the censoring indicators. In other words,

$$
Y_{1 i}=V_{1 i}, \text { if } C_{i}=1 \text { and } Y_{1 i}=V_{2 i}=N A, \text { if } C_{i}=0,
$$

for all $i \in\{1, \ldots, n\}$. Notice that $V_{2 i}=N A$ is equivalent to write $-\infty<V_{2 i}<\infty$.

\subsection{The likelihood function}

To obtain the likelihood function of the SLn model, first note that if $C_{i}=1$, then $Y_{1 i} \sim N\left(\mathbf{x}_{i}^{\top} \boldsymbol{\beta}, \sigma^{2}\right)$ and $Y_{2 i} \mid Y_{1 i}=$ $V_{1 i} \sim N\left(\mu_{c}, \sigma_{c}^{2}\right)$, where

$$
\mu_{c}=\mathbf{w}_{i}^{\top} \gamma+\frac{\rho}{\sigma}\left(V_{1 i}-\mathbf{x}_{i}^{\top} \boldsymbol{\beta}\right), \sigma_{c}^{2}=\left(1-\rho^{2}\right) .
$$

Thus, the contribution in the likelihood is

$$
f\left(Y_{1 i} \mid \boldsymbol{\theta}\right) P\left(Y_{2 i}>0 \mid Y_{1 i}=V_{1 i}\right)=\phi\left(V_{1 i} \mid \mathbf{x}_{i}^{\top} \boldsymbol{\beta}, \sigma^{2}\right) \Phi\left(\frac{\mu_{c}}{\sigma_{c}}\right) .
$$

If $C_{i}=0$, then the contribution in the likelihood is

$$
P\left(Y_{2 i} \leq 0\right)=\Phi\left(-\mathbf{w}_{i}^{\top} \gamma\right)
$$

Therefore, the likelihood function of $\boldsymbol{\theta}=\left(\boldsymbol{\beta}^{\top}, \boldsymbol{\gamma}^{\top}, \sigma^{2}, \rho\right)^{\top}$ is

$$
L(\boldsymbol{\theta} \mid \mathbf{V}, \mathbf{C})=\prod_{i=1}^{n}\left[\phi\left(V_{1 i} \mid \mathbf{x}_{i}^{\top} \boldsymbol{\beta}, \sigma^{2}\right) \Phi\left(\frac{\mu_{c}}{\sigma_{c}}\right)\right]^{C_{i}}\left[\Phi\left(-\mathbf{w}_{i}^{\top} \gamma\right)\right]^{1-C_{i}}
$$

where $\mathbf{V}=\left(\mathbf{V}_{1}, \ldots, \mathbf{V}_{n}\right)$ and $\mathbf{C}=\left(C_{1}, \ldots, C_{n}\right)$. The log-likelihood function for the observed data is given by $\ell(\theta)=\ell(\boldsymbol{\theta} \mid \mathbf{V}, \mathbf{C})=\ln L(\boldsymbol{\theta} \mid \mathbf{V}, \mathbf{C})$,

\subsection{Parameter estimation via the EM algorithm}

We describe in detail how to carry out ML estimation for the SLn model. Let $\mathbf{y}=\left(\mathbf{y}_{1}^{\top}, \ldots, \mathbf{y}_{n}^{\top}\right)^{\top}, \mathbf{V}=\left(\mathbf{V}_{1}, \ldots, \mathbf{V}_{n}\right)$ and $\mathbf{C}=\left(C_{1}, \ldots, C_{n}\right)$, and that we observe $\left(\mathbf{V}_{i}, C_{i}\right)$ for the $i$ th subject, $i \in\{1, \ldots, n\}$. In the estimation procedure, 
$\mathbf{y}$ is treated as hypothetical missing data, and augmented with the observed data set $(\mathbf{C}, \mathbf{V})$, we have $\mathbf{y}_{c}=(\mathbf{C}, \mathbf{V}, \mathbf{y})^{\top}$. Hence, the EM-type algorithm is applied to the complete-data log-likelihood function given by

where

$$
\ell_{c}\left(\boldsymbol{\theta} \mid \mathbf{y}_{c}\right)=\sum_{i=1}^{n} \ell_{i c}(\boldsymbol{\theta})
$$

$$
\ell_{i c}(\boldsymbol{\theta})=-\frac{1}{2}\left\{\ln |\boldsymbol{\Sigma}|+\left(\mathbf{y}_{i}-\boldsymbol{\mu}_{i}\right)^{\top} \boldsymbol{\Sigma}^{-1}\left(\mathbf{y}_{i}-\boldsymbol{\mu}_{i}\right)\right\}+c,
$$

where $c$ is a constant that does not depend on $\boldsymbol{\theta}, \boldsymbol{\mu}_{i}=\mathbf{X}_{i c} \boldsymbol{\beta}_{c}$ and $\boldsymbol{\Sigma}$ as defined in (5). Finally, the EM algorithm for the SLn model can be summarized through the following two steps.

E-step: Given the current estimate $\boldsymbol{\theta}=\widehat{\boldsymbol{\theta}}^{(k)}$ at the $k$ th step of the algorithm, the E-step provides the conditional expectation of the complete data log-likelihood function

$$
Q\left(\boldsymbol{\theta} \mid \widehat{\boldsymbol{\theta}}^{(k)}\right)=\mathrm{E}\left[\ell_{c}\left(\boldsymbol{\theta} \mid \mathbf{y}_{c}\right) \mid \mathbf{V}, \mathbf{C}, \widehat{\boldsymbol{\theta}}^{(k)}\right]=\sum_{i=1}^{n} Q_{i}\left(\boldsymbol{\theta} \mid \widehat{\boldsymbol{\theta}}^{(k)}\right)
$$

where

$$
Q_{i}\left(\boldsymbol{\theta} \mid \widehat{\boldsymbol{\theta}}^{(k)}\right)=Q_{i}\left(\boldsymbol{\beta}, \boldsymbol{\gamma}, \sigma^{2}, \rho \mid \widehat{\boldsymbol{\theta}}^{(k)}\right)=-\frac{1}{2} \ln |\boldsymbol{\Sigma}|-\frac{1}{2} \operatorname{tr}\left[\left\{{\widehat{\mathbf{y}_{i}^{2}}}^{(k)}-\widehat{\mathbf{y}}_{i}^{(k)} \boldsymbol{\mu}_{i}^{\top}-\boldsymbol{\mu}_{i} \widehat{\mathbf{y}}_{i}^{\top(k)}+\boldsymbol{\mu}_{i} \boldsymbol{\mu}_{i}^{\top}\right\} \boldsymbol{\Sigma}^{-1}\right],
$$

with $\widehat{\mathbf{y}}_{i}^{(k)}=\mathrm{E}\left[\mathbf{Y}_{i} \mid \mathbf{V}_{i}, C_{i}, \widehat{\boldsymbol{\theta}}^{(k)}\right]$ and ${\widehat{\mathbf{y}_{i}^{2}}}^{(k)}=\mathrm{E}\left[\mathbf{Y}_{i} \mathbf{Y}_{i}^{\top} \mid \mathbf{V}_{i}, C_{i}, \widehat{\boldsymbol{\theta}}^{(k)}\right]$ and $\operatorname{tr}(\mathbf{A})$ indicates the trace of the matrix $\mathbf{A}$. Following Matos et al. (2013), for $C_{i}=1$ we have

$$
\widehat{\mathbf{y}}_{i}^{(k)}=\left(V_{i}, \hat{w}^{c}{ }_{i}\right)^{\top}, \widehat{\mathbf{y}}_{i}^{(k)}=\left[\begin{array}{cc}
V_{1 i}^{2} & V_{1 i} \hat{w}^{c} \\
V_{1 i}^{2} \hat{w}^{c} i & \hat{w}^{2 c}{ }_{i}
\end{array}\right],
$$

where $w_{i}^{c}=E\left[W_{i} \mid \boldsymbol{\theta}^{(k)}\right], w_{i}^{2 c}=E\left[W_{i}^{2} \mid \boldsymbol{\theta}^{(k)}\right]$, with $W_{i} \sim T N_{1}\left(\mu_{c}, \sigma_{c}^{2} ;(0, \infty)\right)$. For $C_{i}=0$

where $\mathbf{W}_{i} \sim T N_{2}\left(\boldsymbol{\mu}_{i}, \boldsymbol{\Sigma} ; \mathbb{A}\right)$, with

$$
\widehat{\mathbf{y}}_{i}^{(k)}=E\left[\mathbf{W}_{i} \mid \boldsymbol{\theta}^{(k)}\right],{\widehat{\mathbf{y}^{2}}}_{i}^{(k)}=E\left[\mathbf{W}_{i} \mathbf{W}_{i}^{\top} \mid \boldsymbol{\theta}^{(k)}\right]
$$

$$
\mathbb{A}=\left\{\left(x_{1}, x_{2}\right) \in \mathbb{R}^{2}:-\infty \leq x_{1} \leq \infty,-\infty \leq x_{2} \leq 0\right\} .
$$

Here $T N_{p}(\boldsymbol{\mu}, \boldsymbol{\Sigma}, \mathbb{A})$ denotes the p-variate truncated normal distribution with location $\boldsymbol{\mu}$, scale matrix $\boldsymbol{\Sigma}$ over the truncation region $\mathbb{A}$.

M-step: By the invariance property of ML estimators, we use the parameter transformations $\psi=\sigma^{2}\left(1-\rho^{2}\right)$ and $\rho^{*}=\rho \sigma$ in order to get closed form expression. Thus, in this step, $Q\left(\boldsymbol{\theta} \mid \widehat{\boldsymbol{\theta}}^{(k)}\right)$ is conditionally maximized with respect to $\boldsymbol{\theta}$ and a new estimate $\widehat{\boldsymbol{\theta}}^{(k+1)}$ is obtained. Specifically, we have that

$$
\begin{aligned}
\widehat{\boldsymbol{\beta}}_{c}^{(k+1)} & =\left(\sum_{i=1}^{n} \mathbf{X}_{i c}^{\top} \widehat{\boldsymbol{\Sigma}}^{(k)} \mathbf{X}_{i}\right)^{-1} \sum_{i=1}^{n} \mathbf{X}_{i c}^{\top} \widehat{\boldsymbol{\Sigma}}^{(k)} \widehat{\mathbf{y}}_{i}^{(k)}, \\
\widehat{\psi}^{(k+1)} & =\frac{1}{n} \sum_{i=1}^{n}\left(\widehat{\Gamma}_{11 i}^{(k)}-\widehat{\rho}^{*(k)}\left(\widehat{\Gamma}_{12 i}^{(k)}+\widehat{\Gamma}_{21 i}^{(k)}\right)+\widehat{\rho}^{* 2(k)} \widehat{\Gamma}_{22 i}^{(k)}\right), \\
{\widehat{\rho^{*}}}^{(k+1)} & =\frac{\sum_{i=1}^{n}\left(\widehat{\Gamma}_{12 i}^{(k)}+\widehat{\Gamma}_{21 i}^{(k)}\right)}{2 \sum_{i=1}^{n} \widehat{\Gamma}_{22 i}^{(k)}},
\end{aligned}
$$

where $\widehat{\Gamma}_{k l i}^{(k)}$ is the $k l$ th element of the matrix $\widehat{\boldsymbol{\Gamma}}_{i}^{(k)}=\left\{{\widehat{\mathbf{y}_{i}^{2}}}^{(k)}-\widehat{\mathbf{y}}_{i}^{(k)} \widehat{\boldsymbol{\mu}}_{i}^{(k) \top}-\widehat{\boldsymbol{\mu}}_{i}^{(k)}\left(\widehat{\mathbf{y}}_{i}^{(k)}\right)^{\top}+{\widehat{\boldsymbol{\mu}_{i}}}^{(k)} \widehat{\boldsymbol{\mu}}_{i}^{(k) \top}\right\}$. The algorithm is terminated when the relative distance between two successive evaluations of the log-likelihood defined in 99 is less than a tolerance, i.e., $\left|\ell\left(\widehat{\boldsymbol{\theta}}^{(k+1)} \mid \mathbf{V}, \mathbf{C}\right) / \ell\left(\widehat{\boldsymbol{\theta}}^{(k)} \mid \mathbf{V}, \mathbf{C}\right)-1\right|<\epsilon$, for example, $\epsilon=10^{-6}$. Once converged, we can recover $\widehat{\sigma}^{2}$ and $\widehat{\rho}$ using the expressions

$$
\widehat{\sigma}^{2}=\widehat{\psi}+{\widehat{\rho^{*}}}^{2} \quad \text { and } \quad \widehat{\rho}=\frac{\widehat{\rho^{*}}}{\widehat{\sigma}} .
$$

The initial values of the parameters for the EM algorithm are obtained from the 2-step procedure, which are obtained from the R package sampleselection (Henningsen et al., 2019). 


\subsection{Provision of standard errors}

In this section, we describe how to obtain the standard errors of the ML estimates for the SLn model. We follow the information-based method exploited by Basford et al. (1997) to compute the asymptotic covariance of the ML estimates. The empirical information matrix, according to Meilijson (1989)'s formula, is defined as

$$
\mathbf{I}_{e}(\boldsymbol{\theta} \mid \mathbf{y})=\sum_{i=1}^{n} s\left(\mathbf{y}_{i} \mid \boldsymbol{\theta}\right) s^{\top}\left(\mathbf{y}_{i} \mid \boldsymbol{\theta}\right)-\frac{1}{n} S\left(\mathbf{y}_{i} \mid \boldsymbol{\theta}\right) S^{\top}\left(\mathbf{y}_{i} \mid \boldsymbol{\theta}\right),
$$

where $S\left(\mathbf{y}_{i} \mid \boldsymbol{\theta}\right)=\sum_{i=1}^{N} s\left(\mathbf{y}_{i} \mid \boldsymbol{\theta}\right)$ and $s\left(\mathbf{y}_{i} \mid \boldsymbol{\theta}\right)$ is the empirical score function for the $i$ th subject. It is noted from the result of Louis (1982) that the individual score can be determined as

$$
s\left(\mathbf{y}_{i} \mid \boldsymbol{\theta}\right)=\mathrm{E}\left[\frac{\partial \ell_{i c}(\boldsymbol{\theta})}{\partial \boldsymbol{\theta}} \mid \mathbf{V}_{i}, C_{i}, \boldsymbol{\theta}\right] .
$$

Using the ML estimates $\widehat{\boldsymbol{\theta}}$ in $s\left(\mathbf{y}_{i} \mid \boldsymbol{\theta}\right)$, leads to $S\left(\mathbf{y}_{i} \mid \widehat{\boldsymbol{\theta}}\right)=0$, so from 14 we have that

$$
\mathbf{I}_{e}(\widehat{\boldsymbol{\theta}} \mid \mathbf{y})=\sum_{i=1}^{n} \widehat{\mathbf{s}}_{i} \widehat{\mathbf{s}}_{i}^{\top}
$$

where $\widehat{\mathbf{s}}_{i}$ is an individual score vector given by $\widehat{\mathbf{s}}_{i}=\left(\widehat{s}_{i, \boldsymbol{\beta}_{c}}, \widehat{s}_{i, \sigma}, \widehat{s}_{i, \rho}\right)$. So, the expressions for the elements of $\widehat{\mathbf{s}}_{i}$ are given by:

$$
\begin{aligned}
\widehat{s}_{i, \boldsymbol{\beta}_{c}} & =\frac{1}{2} \mathbf{X}_{i c}^{\top} \boldsymbol{\Sigma}^{-1} \widehat{\mathbf{y}}_{i}+\frac{1}{2} \widehat{\mathbf{y}}_{i}^{\top} \boldsymbol{\Sigma}^{-1} \mathbf{X}_{i c}-\mathbf{X}_{i c}^{\top} \boldsymbol{\Sigma}^{-1} \mathbf{X}_{i c} \boldsymbol{\beta}_{c} \\
\widehat{s}_{i, \sigma} & =-\frac{1}{2} \operatorname{tr}\left(\boldsymbol{\Sigma}^{-1} \mathbf{B}\right)+\frac{1}{2} \operatorname{tr}\left(\boldsymbol{\Gamma}_{i} \boldsymbol{\Sigma}^{-1} \mathbf{B} \Sigma^{-1}\right) \\
\widehat{s}_{i, \rho} & =-\frac{1}{2} \operatorname{tr}\left(\boldsymbol{\Sigma}^{-1} \mathbf{D}\right)+\frac{1}{2} \operatorname{tr}\left(\boldsymbol{\Gamma}_{i} \boldsymbol{\Sigma}^{-1} \mathbf{D} \boldsymbol{\Sigma}^{-1}\right)
\end{aligned}
$$

where $\boldsymbol{\Gamma}_{i}$ is as defined in $111--13, \boldsymbol{\beta}_{c}$ and $\mathbf{X}_{i c}$ as defined in $\left[7, \mathbf{B}=\left[\begin{array}{cc}2 \sigma & \rho \\ \rho & 0\end{array}\right]\right.$, and $\mathbf{D}=\left[\begin{array}{ll}0 & \sigma \\ \sigma & 0\end{array}\right]$.

The SLn model discussed in this section was criticized in the literature because of its sensitivity to the normality assumption. In the next section, we establish a new link between the SL model and the Student's-t distribution, called the Heckman selection-t model (SLt), as introduced by Marchenko and Genton (2012).

\section{The Heckman selection-t model}

In order to accommodate for heavy-tailedness, Marchenko and Genton (2012) proposed the SLt model, replacing the normal assumption of error terms in 5 by a bivariate Student'-t distribution with an unknown number of degrees of freedom $\nu$ :

$$
\left[\begin{array}{l}
\epsilon_{1 i} \\
\epsilon_{2 i}
\end{array}\right] \sim t_{2}\left(\left[\begin{array}{l}
0 \\
0
\end{array}\right], \boldsymbol{\Sigma}=\left[\begin{array}{cc}
\sigma^{2} & \rho \sigma \\
\rho \sigma & 1
\end{array}\right], \nu\right)
$$

As in the SLn model, and ignoring censoring for the moment, we can represent the robust SLt model belong to a censored data framework, as follows:

$$
\mathbf{Y}_{1}, \ldots, \mathbf{Y}_{n} \stackrel{\text { ind. }}{\sim} t_{2}\left(\boldsymbol{\mu}_{i}, \boldsymbol{\Sigma}, \nu\right)
$$

where for each $i \in\{1, \ldots, n\}, \mathbf{Y}_{i}=\left(Y_{i 1}, Y_{i 2}\right)^{\top}$ is the vector of independent responses for sample unit $i, \boldsymbol{\mu}_{i}=\mathbf{X}_{i c} \boldsymbol{\beta}_{c}$ with $\mathbf{X}_{i c}$ and $\boldsymbol{\beta}_{c}$ as defined in (7). We consider the approach proposed by Vaida and Liu (2009), Matos et al. (2013) and Matos et al. (2013) to represent the model belong to the structure of a censored linear model, where the observed data for the $i$ th subject is given by $\left(\mathbf{V}_{i}, C_{i}\right)$, where $\mathbf{V}_{i}$ represents the vector of censored readings and $C_{i}=I_{\left\{Y_{2 i}>0\right\}}$ is the censoring indicators. The model defined in (3), 4), 8) and (17) is henceforth called the SLt model.

\subsection{The likelihood function}

To obtain the likelihood function of the SLt model, first from Proposition 1 note that if $C_{i}=1$, then $Y_{1 i} \sim t\left(\mathbf{x}_{i}^{\top} \boldsymbol{\beta}, \sigma^{2}, \nu\right)$ and $Y_{2 i} \mid Y_{1 i}=V_{1 i} \sim t\left(\mu_{t i}, \sigma_{t i}^{2}, \nu+1\right)$, where

$$
\mu_{t i}=\mathbf{w}_{i}^{\top} \boldsymbol{\gamma}+\frac{\rho}{\sigma}\left(V_{1 i}-\mathbf{x}_{i}^{\top} \boldsymbol{\beta}\right), \sigma_{t i}^{2}=\frac{\nu+\delta\left(V_{1 i}\right)}{\nu+1}\left(1-\rho^{2}\right)
$$


where $\delta\left(V_{i}\right)=\frac{\left(V_{1 i}-\mathbf{x}_{i}^{\top} \boldsymbol{\beta}\right)^{2}}{\sigma^{2}}$. Thus, the contribution in the likelihood function of $\boldsymbol{\theta}=\left(\boldsymbol{\beta}^{\top}, \boldsymbol{\gamma}^{\top}, \sigma^{2}, \rho, \nu\right)^{\top}$, given the observed sample $(\mathbf{V}, \mathbf{C})$, is

$$
f\left(Y_{1 i} \mid \boldsymbol{\theta}\right) P\left(Y_{2 i}>0 \mid Y_{1 i}=V_{i}\right)=t\left(V_{1 i} \mid \mathbf{x}_{i}^{\top} \boldsymbol{\beta}, \sigma^{2}, \nu\right) T\left(-\infty, 0 ;-\mu_{t i}, \sigma_{t i}^{2}, \nu+1\right) .
$$

If $C_{i}=0$, then the contribution in the likelihood function is

$$
P\left(Y_{2 i} \leq 0\right)=T\left(-\infty, 0 ; \mathbf{w}_{i}^{\top} \boldsymbol{\gamma}, 1, \nu\right) .
$$

Therefore, the likelihood function of $\boldsymbol{\theta}=\left(\boldsymbol{\beta}^{\top}, \boldsymbol{\gamma}^{\top}, \sigma^{2}, \rho, \nu\right)^{\top}$, given the observed sample $(\mathbf{V}, \mathbf{C})$, is

$$
L(\boldsymbol{\theta} \mid \mathbf{V}, \mathbf{C})=\prod_{i=1}^{n}\left[t\left(V_{1 i} \mid \mathbf{x}_{i}^{\top} \boldsymbol{\beta}, \sigma^{2}, \nu\right) T\left(-\infty, 0 ;-\mu_{t i}, \sigma_{t i}^{2}, \nu+1\right)\right]^{C_{i}}\left[T\left(-\infty, 0 ; \mathbf{w}_{i}^{\top} \boldsymbol{\gamma}, 1, \nu\right)\right]^{1-C_{i}},
$$

where $\mathbf{V}=\left(\mathbf{V}_{1}, \ldots, \mathbf{V}_{n}\right)$ and $\mathbf{C}=\left(C_{1}, \ldots, C_{n}\right)$. The log-likelihood function for the observed data is given by $\ell(\theta)=\ell(\boldsymbol{\theta} \mid$ $\mathbf{V}, \mathbf{C})=\ln L(\boldsymbol{\theta} \mid \mathbf{V}, \mathbf{C})$, that is,

$$
\ell(\boldsymbol{\theta})=\sum_{i=1}^{n}\left[C_{i} \ln t\left(V_{1 i} \mid \mathbf{x}_{i}^{\top} \boldsymbol{\beta}, \sigma^{2}, \nu\right)+C_{i} \ln T\left(-\infty, 0 ;-\mu_{t i}, \sigma_{t i}^{2}, \nu+1\right)\right]+\sum_{i=1}^{n}\left(1-C_{i}\right) \ln \left[T\left(-\infty, 0 ; \mathbf{w}_{i}^{\top} \boldsymbol{\gamma}, 1, \nu\right)\right]
$$

The ML estimate $\widehat{\boldsymbol{\theta}}$ of the vector of unknown parameters can be calculated by maximizing the log-likelihood given in 19 . There are many optimization procedures available in standard programs, such as the opt im routine in $\mathrm{R}$, which need only the original estimator function. A disadvantage of direct maximization of the log-likelihood function is that it may not converge unless good starting values are used. Thus, we also propose the EM algorithm for parameter estimation, which is stable and straightforward to implement since the iterations converge monotonically and no second derivatives are required. Moreover, the EM estimates are quite insensitive to the starting values, as discussed by Zhao et al. (2020) regarding the SLn model.

\subsection{Parameter estimation via the EM algorithm}

Note first that by using the representation (2) and ignoring censoring for the moment, we have that the distribution of $\mathbf{Y}_{i}$ can be hierarchically written as

$$
\mathbf{Y}_{i} \mid U_{i}=u_{i} \stackrel{\text { ind. }}{\sim} \mathcal{N}_{2}\left(\boldsymbol{\mu}_{i}, u_{i}^{-1} \boldsymbol{\Sigma}\right), \quad U_{i} \stackrel{\text { ind. }}{\sim} \mathcal{G}(\nu / 2, \nu / 2)
$$

Let $\mathbf{y}=\left(\mathbf{y}_{1}^{\top}, \ldots, \mathbf{y}_{n}^{\top}\right)^{\top}, \mathbf{V}=\left(\mathbf{V}_{1}, \ldots, \mathbf{V}_{n}\right), \mathbf{C}=\left(C_{1}, \ldots, C_{n}\right), \mathbf{u}=\left(U_{1}, \ldots, U_{n}\right)$, and that we observe $\left(\mathbf{V}_{i}, C_{i}\right)$ for the $i$ th subject. In the estimation procedure, $\mathbf{y}$ and $\mathbf{u}$ are treated as hypothetical missing data, and augmented with the observed data set we have $\mathbf{y}_{c}=(\mathbf{C}, \mathbf{V}, \mathbf{y}, \mathbf{u})^{\top}$. Hence, the EM-type algorithm is applied to the complete-data log-likelihood function given by

$$
\ell_{c}\left(\boldsymbol{\theta} \mid \mathbf{y}_{c}\right)=\sum_{i=1}^{n} \ell_{i c}(\boldsymbol{\theta})
$$

where

$$
\ell_{i c}(\boldsymbol{\theta})=-\frac{1}{2}\left\{\ln |\boldsymbol{\Sigma}|+u_{i}\left(\mathbf{y}_{i}-\boldsymbol{\mu}_{i}\right)^{\top} \boldsymbol{\Sigma}^{-1}\left(\mathbf{y}_{i}-\boldsymbol{\mu}_{i}\right)\right\}+\ln h\left(u_{i} \mid \nu\right)+c,
$$

where $c$ is a constant that does not depend on $\boldsymbol{\theta}$ and $h\left(u_{i} \mid \nu\right)$ is the $\operatorname{Gamma}(\nu / 2, \nu / 2)$ pdf. The EM algorithm for the SLt model can be summarized through the following two steps.

E-step: Given the current estimate $\boldsymbol{\theta}=\widehat{\boldsymbol{\theta}}^{(k)}$ at the $k$ th step of the algorithm, the E-step provides the conditional expectation of the complete data $\log$-likelihood function

$$
Q\left(\boldsymbol{\theta} \mid \widehat{\boldsymbol{\theta}}^{(k)}\right)=\mathrm{E}\left[\ell_{c}\left(\boldsymbol{\theta} \mid \mathbf{y}_{c}\right) \mid \mathbf{V}, \mathbf{C}, \widehat{\boldsymbol{\theta}}^{(k)}\right]=\sum_{i=1}^{n} Q_{i}\left(\boldsymbol{\theta} \mid \widehat{\boldsymbol{\theta}}^{(k)}\right),
$$

where

$Q_{i}\left(\boldsymbol{\theta} \mid \widehat{\boldsymbol{\theta}}^{(k)}\right)=Q_{i}\left(\boldsymbol{\beta}^{\top}, \boldsymbol{\gamma}^{\top}, \sigma^{2}, \rho, \nu \mid \widehat{\boldsymbol{\theta}}^{(k)}\right)=-\frac{1}{2} \ln |\boldsymbol{\Sigma}|-\frac{1}{2} \operatorname{tr}\left[\left\{{\widehat{u \mathbf{y}_{i}^{2}}}^{(k)}-\widehat{u \mathbf{y}}_{i}^{(k)} \boldsymbol{\mu}_{i}^{\top}-\boldsymbol{\mu}_{i}\left(\widehat{u \mathbf{y}}_{i}^{(k)}\right)^{\top}+\widehat{u}_{i}^{(k)} \boldsymbol{\mu}_{i} \boldsymbol{\mu}_{i}^{\top}\right\} \boldsymbol{\Sigma}^{-1}\right]$,

with $\widehat{u \mathbf{y}}_{i}^{(k)}=\mathrm{E}\left[U_{i} \mathbf{Y}_{i} \mid \mathbf{V}_{i}, \mathbf{C}_{i}, \widehat{\boldsymbol{\theta}}^{(k)}\right],{\widehat{u \mathbf{y}_{i}^{2}}}^{(k)}=\mathrm{E}\left[U_{i} \mathbf{Y}_{i} \mathbf{Y}_{i}^{\top} \mid \mathbf{V}_{i}, \mathbf{C}_{i}, \widehat{\boldsymbol{\theta}}^{(k)}\right]$ and $\widehat{u}_{i}^{(k)}=\mathrm{E}\left[U_{i} \mid \mathbf{V}_{i}, \mathbf{C}_{i}, \widehat{\boldsymbol{\theta}}^{(k)}\right]$. Note that $\widehat{\kappa}_{i}^{(k)}=\mathrm{E}\left[\ln h\left(U_{i} \mid \nu\right) \mid \mathbf{V}, \mathbf{C}, \widehat{\boldsymbol{\theta}}^{(k)}\right]$ is analytically intractable. Instead, we avoid the calculation of $\widehat{\kappa}_{i}^{(k)}$ by performing the CMLstep for updating $\nu$. As in the normal case, we use the parameter transformations $\psi=\sigma^{2}\left(1-\rho^{2}\right)$ and $\rho^{*}=\rho \sigma$ in order to get closed form expression in the M-Step. 
A PREPRINT - JUNE 16, 2020

M-step: In this step, $Q\left(\boldsymbol{\theta} \mid \widehat{\boldsymbol{\theta}}^{(k)}\right)$ is conditionally maximized with respect to $\boldsymbol{\beta}_{c}, \sigma^{2}, \rho$ and a new estimate $\widehat{\boldsymbol{\beta}}_{c}^{(k+1)}, \widehat{\boldsymbol{\sigma}}^{2(k+1)}, \widehat{\rho}^{(k+1)}$ is obtained. Specifically, we have that

$$
\begin{aligned}
\widehat{\boldsymbol{\beta}}_{c}^{(k+1)} & =\left(\sum_{i=1}^{n} \widehat{u}_{i}^{(k)} \mathbf{X}_{i c}^{\top} \boldsymbol{\Sigma}^{(k)} \mathbf{X}_{i c}\right)^{-1} \sum_{i=1}^{n} \mathbf{X}_{i c}^{\top} \boldsymbol{\Sigma}^{(k)} \widehat{u \mathbf{y}}_{i}^{(k)}, \widehat{\boldsymbol{\mu}}_{i}^{(k+1)}=\mathbf{X}_{i c} \widehat{\boldsymbol{\beta}}_{c}^{(k+1)} \\
\widehat{\psi}^{(k+1)} & =\frac{1}{n} \sum_{i=1}^{n}\left(\widehat{\Gamma}_{11 i}^{(k)}-\widehat{\rho}^{*(k)}\left(\widehat{\Gamma}_{12 i}^{(k)}+\widehat{\Gamma}_{21 i}^{(k)}\right)+\widehat{\rho}^{* 2(k)} \widehat{\Gamma}_{22 i}^{(k)}\right), \\
\widehat{\rho}^{*}(k+1) & =\frac{\sum_{i=1}^{n}\left(\widehat{\Gamma}_{12 i}^{(k)}+\widehat{\Gamma}_{21 i}^{(k)}\right)}{2 \sum_{i=1}^{n} \widehat{\Gamma}_{22 i}^{(k)}}, \\
\widehat{\sigma}^{(k+1)} & =\widehat{\psi}^{(k+1)}+{\widehat{\rho^{*}}}^{2(k+1)} \quad \text { and } \quad \widehat{\rho}^{(k+1)}=\frac{\widehat{\rho}^{*(k+1)}}{\widehat{\sigma}^{(k+1)}} .
\end{aligned}
$$

where $\widehat{\Gamma}_{k l i}^{(k)}$ is the $k$ lth element of the matrix $\widehat{\boldsymbol{\Gamma}}_{i}^{(k)}=\left\{{\widehat{u \mathbf{y}_{i}^{2}}}^{(k)}-\widehat{u \mathbf{y}}_{i}^{(k)}{\widehat{\boldsymbol{\mu}_{i}}}^{(k) \top}-{\widehat{\boldsymbol{\mu}_{i}}}^{(k)}\left(\widehat{\mathbf{y}}_{i}^{(k)}\right)^{\top}+\widehat{u}_{i}^{(k)}{\widehat{\boldsymbol{\mu}_{i}}}^{(k)}{\widehat{\boldsymbol{\mu}_{i}}}^{(k) \top}\right\}$.

CLM-step: Update $\widehat{\nu}^{(k+1)}$ by maximizing the actual marginal log-likelihood function, obtaining

$$
\begin{aligned}
\widehat{\nu}^{(k+1)}= & \operatorname{argmax}_{\nu}\left\{\sum_{i=1}^{n} C_{i} \ln \left[t\left(V_{1 i} \mid \mathbf{x}_{i}^{\top} \widehat{\boldsymbol{\beta}}^{(k+1)}, \widehat{\sigma}^{2(k+1)}, \nu\right) T\left(-\infty, 0 ;-\widehat{\mu}_{t i}^{(k+1)}, \widehat{\sigma}_{t i}^{2(k+1)}, \nu+1\right)\right]\right. \\
& \left.+\sum_{i=1}^{n}\left(1-C_{i}\right) \ln T\left(-\infty, 0 ; \mathbf{w}_{i}^{\top} \widehat{\boldsymbol{\gamma}}^{(k+1)}, 1, \nu\right)\right\} .
\end{aligned}
$$

The algorithm is iterated until a suitable convergence rule is satisfied. It is important to stress that, from Eqs. 21] - 24], the E-step reduces to the computation of $\widehat{u \mathbf{y}_{i}^{2}}, \widehat{u \mathbf{y}}_{i}$, and $\widehat{u}_{i}$. To compute these expected values, first observe that they can be written in terms of $\mathrm{E}\left(U_{i} \mid \mathbf{Y}_{i}\right)$, where $\mathbf{Y}_{i} \sim t_{p}(\boldsymbol{\mu}, \boldsymbol{\Sigma}, \nu)$ - see the definition of $U_{i}$ in 20 .

For example, we have that $\widehat{u}_{i}=\mathrm{E}\left[\mathrm{E}\left(U_{i} \mid \mathbf{Y}_{i}\right) \mid \mathbf{V}_{i}, \mathbf{C}_{i}, \widehat{\boldsymbol{\theta}}^{(k)}\right]$. It is straightforward to prove that $\mathrm{E}\left[U_{i} \mid \mathbf{Y}_{i}\right]=(\nu+1) /(\nu+\delta)$, where $\delta=\left(\mathbf{Y}_{i}-\boldsymbol{\mu}_{i}\right)^{\top} \boldsymbol{\Sigma}^{-1}\left(\mathbf{Y}_{i}-\boldsymbol{\mu}_{i}\right)$. Then, we can use Propositions 3 and 4 to obtain closed form expressions as follows:

1. If $C_{i}=0$, from Proposition 3, we have

where

$$
\begin{aligned}
{\widehat{u \mathbf{y}_{i}^{2}}}^{(k)} & =\mathrm{E}\left[U_{i} \mathbf{Y}_{i} \mathbf{Y}_{i}^{\top} \mid \mathbf{V}_{i}, C_{i}, \widehat{\boldsymbol{\theta}}^{(k)}\right]=\widehat{\varphi}^{(k)}\left(\mathbf{V}_{i}\right) \widehat{\mathbf{w}}_{i}^{2^{c(k)}} \\
\widehat{u \mathbf{y}}_{i}^{(k)} & =\mathrm{E}\left[U_{i} \mathbf{Y}_{i} \mid \mathbf{V}_{i}, C_{i}, \widehat{\boldsymbol{\theta}}^{(k)}\right]=\widehat{\varphi}^{(k)}\left(\mathbf{V}_{i}\right) \widehat{\mathbf{w}}_{i}^{c(k)} \\
\widehat{u}_{i}^{(k)} & =\mathrm{E}\left[U_{i} \mid \mathbf{V}_{i}, C_{i}, \widehat{\boldsymbol{\theta}}^{(k)}\right]=\widehat{\varphi}^{(k)}\left(\mathbf{V}_{i}\right)
\end{aligned}
$$

$$
\begin{gathered}
\widehat{\varphi}^{(k)}\left(\mathbf{V}_{i}\right)=\frac{T_{2}\left((-\infty,-\infty),(\infty, 0) ; \widehat{\boldsymbol{\mu}}_{i}^{(k)}, \quad \widehat{\boldsymbol{\Sigma}}^{*(k)}, \nu+2\right)}{T_{2}\left((-\infty,-\infty),(\infty, 0) ; \widehat{\boldsymbol{\mu}}^{(k)}, \quad \widehat{\boldsymbol{\Sigma}}^{(k)}, \nu\right)}, \quad \widehat{\mathbf{W}}_{i}^{c(k)}=\mathrm{E}\left[\mathbf{W}_{i} \mid \widehat{\boldsymbol{\theta}}^{(k)}\right], \quad \widehat{\mathbf{W}}_{i}^{c(k)}=\mathrm{E}\left[\mathbf{W}_{i} \mathbf{W}_{i}^{\top} \mid \widehat{\boldsymbol{\theta}}^{(k)}\right], \\
\mathbf{W}_{i} \sim T t_{2}\left(\widehat{\boldsymbol{\mu}}^{(k)}, \widehat{\boldsymbol{\Sigma}}^{*(k)}, \nu+2 ; \mathbb{A}\right), \quad \widehat{\boldsymbol{\Sigma}}^{*(k)}=\frac{\nu}{\nu+2} \widehat{\boldsymbol{\Sigma}}^{(k)},
\end{gathered}
$$

with $\mathbb{A}_{i}$ as defined in 10 . To compute $\mathrm{E}\left[\mathbf{W}_{i}\right]$ and $\mathrm{E}\left[\mathbf{W}_{i} \mathbf{W}_{i}^{\top}\right]$ we use the R package MomTrunc Galarza et al. 2020).

2. If $C_{i}=1$, then from Proposition 4 we have that

where

$$
\begin{aligned}
{\widehat{u \mathbf{y}_{i}^{2}}}^{(k)} & =\mathrm{E}\left[U_{i} \mathbf{Y}_{i} \mathbf{Y}_{i}^{\top} \mid Y_{1 i}, \mathbf{V}_{i}, C_{i}, \widehat{\boldsymbol{\theta}}^{(k)}\right]=\left(\begin{array}{cc}
V_{1 i}^{2} \widehat{u}_{i}^{(k)} & \widehat{u}_{i}^{(k)} \widehat{w}_{i}^{c(k) \top} V_{1 i} \\
\widehat{u}_{i}^{(k)} \widehat{w}_{i}^{(k)} V_{1 i} & \widehat{u}_{i}^{(k)} \widehat{w}_{i}^{2^{c(k)}}
\end{array}\right), \\
\widehat{u \mathbf{y}}_{i}^{(k)} & =\mathrm{E}\left[U_{i} \mathbf{Y}_{i} \mid Y_{1 i}, \mathbf{V}_{i}, \mathbf{C}_{i}, \widehat{\boldsymbol{\theta}}^{(k)}\right]=\operatorname{vec}\left(V_{1 i} \widehat{u}_{i}^{(k)}, \widehat{u}_{i}^{(k)} \widehat{w}_{i}^{c(k)}\right), \\
\widehat{u}_{i}^{(k)} & =\mathrm{E}\left[U_{i} \mid Y_{1 i}, \mathbf{V}_{i}, \mathbf{C}_{i}, \widehat{\boldsymbol{\theta}}^{(k)}\right]=\left\{\frac{\nu^{(k)}+1}{\nu^{(k)}+\widehat{\delta}^{(k)}\left(V_{1 i}\right)}\right\} \frac{T\left(0, \infty ; \widehat{\mu}_{t i}^{(k)}, \widetilde{S}_{i}^{(k)}, \nu^{(k)}+3\right)}{T\left(0, \infty ; \widehat{\mu}_{t i}^{(k)}, \hat{\sigma}_{t i}^{2(k)}, \nu^{(k)}+1\right)},
\end{aligned}
$$

$$
\begin{gathered}
\hat{\mu}_{t i}^{(k)}=\mathbf{w}_{i}^{\top} \hat{\gamma}^{(k)}+\frac{\hat{\rho}^{(k)}}{\hat{\sigma}^{(k)}}\left(V_{i}-\mathbf{x}_{i}^{\top} \hat{\boldsymbol{\beta}}^{(k)}\right), \widetilde{S}_{i}^{(k)}=\left\{\frac{\nu^{(k)}+\widehat{\delta}^{(k)}\left(V_{1 i}\right)}{\nu^{(k)}+3}\right\}\left(1-\hat{\rho}^{2}(k)\right), \quad \widehat{\delta}^{(k)}\left(V_{1 i}\right)=\frac{\left(V_{i}-\mathbf{x}_{i}^{\top} \hat{\boldsymbol{\beta}}^{(k)}\right)}{\hat{\sigma}^{(k)}}, \\
\hat{\sigma}_{t i}^{2(k)}=\frac{\nu^{(k)}+\hat{\delta}^{(k)}\left(V_{1 i}\right)}{\nu^{(k)}+1}\left(1-\hat{\rho}^{2(k)}\right),
\end{gathered}
$$

with $W_{i} \sim T t\left(\widehat{\mu}_{t i}^{(k)}, \widetilde{S}_{i}^{(k)}, \nu+3 ;[0, \infty)\right)$. Again, to compute $\widehat{w}_{i}^{c(k)}=\mathrm{E}\left[W_{i}\right]$ and $\widehat{w}_{i}^{c(k)}=\mathrm{E}\left[W_{i}^{2}\right]$ we use the R package MomTrunc. 


\subsection{Provision of standard errors}

To obtain the standard errors of the ML estimates for the SLt model, we use the same strategy discussed in Subsection 3.3 for the SLn model. It is important to stress that the standard error of $\nu$ depends heavily on the calculation of $\widehat{\kappa}_{i}=\mathrm{E}\left[\ln h\left(U_{i} \mid \nu\right) \mid \mathbf{V}, \mathbf{C}, \widehat{\boldsymbol{\theta}}\right]$, which relies on computationally intensive Monte Carlo integration. In our analysis, we focus solely on comparing the standard errors of $\boldsymbol{\beta}_{c}=\left(\boldsymbol{\beta}^{\top}, \boldsymbol{\gamma}^{\top}\right)^{\top}, \sigma$ and $\rho$. So, the expressions for the elements of $\widehat{\mathbf{s}}_{i}$ are given by:

$$
\begin{aligned}
\widehat{s}_{i, \boldsymbol{\beta}_{c}} & =\frac{1}{2} \mathbf{X}_{i c}^{\top} \boldsymbol{\Sigma}^{-1} \widehat{u \mathbf{y}_{i}}+\frac{1}{2}{\widehat{u \mathbf{y}_{i}}}^{\top} \boldsymbol{\Sigma}^{-1} \mathbf{X}_{i c}-\widehat{u}_{i} \mathbf{X}_{i c}^{\top} \boldsymbol{\Sigma}^{-1} \mathbf{X}_{i c} \boldsymbol{\beta}_{c}, \\
\widehat{s}_{i, \sigma} & =-\frac{1}{2} \operatorname{tr}\left(\boldsymbol{\Sigma}^{-1} \mathbf{B}\right)+\frac{1}{2} \operatorname{tr}\left(\boldsymbol{\Gamma}_{i} \boldsymbol{\Sigma}^{-1} \mathbf{B} \Sigma^{-1}\right) \\
\widehat{s}_{i, \rho} & =-\frac{1}{2} \operatorname{tr}\left(\boldsymbol{\Sigma}^{-1} \mathbf{D}\right)+\frac{1}{2} \operatorname{tr}\left(\boldsymbol{\Gamma}_{i} \boldsymbol{\Sigma}^{-1} \mathbf{D} \boldsymbol{\Sigma}^{-1}\right)
\end{aligned}
$$

where $\boldsymbol{\Gamma}_{i}$ as in 21)-24, $\mathbf{B}$ and $\mathbf{D}$ like defined in Subsection 3.3

Table 1: Simulation study 1. Mean estimates (EM), mean standard errors (SE) and Monte Carlo standard error (MC $\mathrm{SE})$ of the 500 Monte Carlo replicates for the data generated from the Normal distribution. The mean AIC and BIC are reported for the SLn and SLt models.

\begin{tabular}{cccccccc}
\hline Sample Size & & \multicolumn{3}{c}{ SLn } & & SLt \\
\cline { 2 - 7 } & TRUE & EM & SE & MC SE & EM & SE & MC SE \\
\hline \multirow{2}{*}{$\beta_{0}=1.000$} & 1.017 & 0.147 & 0.130 & 1.020 & 0.145 & 0.128 \\
& $\beta_{1}=0.500$ & 0.497 & 0.133 & 0.134 & 0.495 & 0.133 & 0.133 \\
& $\gamma_{0}=0.674$ & 0.689 & 0.092 & 0.094 & 0.697 & 0.094 & 0.096 \\
& $\gamma_{1}=0.300$ & 0.316 & 0.157 & 0.157 & 0.320 & 0.159 & 0.160 \\
& $\gamma_{2}=-0.500$ & -0.512 & 0.096 & 0.095 & -0.521 & 0.099 & 0.097 \\
& $\sigma^{2}=1.000$ & 1.004 & 0.085 & 0.081 & 0.983 & 0.089 & 0.081 \\
& $\rho=0.600$ & 0.550 & 0.270 & 0.216 & 0.547 & 0.264 & 0.218 \\
& $\nu=+\infty$ & & & & 111.899 & & \\
AIC & & 736.672 & & & 736.455 & \\
BIC & & 740.193 & & & 739.977 & \\
\hline 500 & $\beta_{0}=1.000$ & 1.006 & 0.090 & 0.092 & 1.009 & 0.090 & 0.091 \\
& $\beta_{1}=0.500$ & 0.505 & 0.088 & 0.092 & 0.503 & 0.088 & 0.092 \\
& $\gamma_{0}=0.674$ & 0.681 & 0.066 & 0.066 & 0.687 & 0.067 & 0.067 \\
& $\gamma_{1}=0.300$ & 0.304 & 0.108 & 0.111 & 0.308 & 0.109 & 0.112 \\
& $\gamma_{2}=-0.500$ & -0.504 & 0.064 & 0.068 & -0.511 & 0.065 & 0.069 \\
& $\sigma^{2}=1.000$ & 1.000 & 0.057 & 0.057 & 0.985 & 0.059 & 0.058 \\
& $\rho=0.600$ & 0.584 & 0.153 & 0.152 & 0.580 & 0.153 & 0.153 \\
$\nu=+\infty$ & & & & 118.592 & & \\
& AIC & & 1480.419 & & & 1480.244 & \\
BIC & & 1484.633 & & & 1484.459 & \\
\hline$\beta_{0}=1.000$ & 1.004 & 0.064 & 0.064 & 1.007 & 0.062 & 0.064 \\
& $\beta_{1}=0.500$ & 0.500 & 0.065 & 0.065 & 0.499 & 0.065 & 0.065 \\
$\gamma_{0}=0.674$ & 0.680 & 0.045 & 0.046 & 0.685 & 0.046 & 0.047 \\
$\gamma_{1}=0.300$ & 0.304 & 0.078 & 0.078 & 0.307 & 0.079 & 0.078 \\
$\gamma_{2}=-0.500$ & -0.503 & 0.047 & 0.049 & -0.508 & 0.048 & 0.049 \\
$\sigma^{2}=1.000$ & 1.000 & 0.041 & 0.041 & 0.987 & 0.042 & 0.041 \\
& $\rho=0.600$ & 0.593 & 0.107 & 0.106 & 0.589 & 0.105 & 0.107 \\
$\nu=+\infty$ & & & & 117.861 & & \\
& AIC & & 2974.585 & & & 2974.495 & \\
BIC & & 2979.493 & & & 2979.403 & \\
\hline 1000 & & & & & & &
\end{tabular}

\section{Simulation study}

In this section, we present three simulations studies. In the first one, we study the finite sample properties of the EM estimates as well as how the robustness of the parameter estimation for the SLt model performs in comparison to model the SLn in the presence 
of model misspecification. The other two studies focus on the vulnerability of the SLn model when data have heavy-tail and varying the correlation parameter $\rho$ and percentage of missing. For each scenario 500 Monte Carlo samples were generated.

\subsection{Finite sample properties}

The initial goal of this simulation is to show the capacity of the proposed EM algorithm to correctly recover the parameters in SL models. Moreover, we test the robustness of the SL alternatives generating data from the Slash distribution.

Table 2: Simulation study 1. Mean estimates (EM), mean standard errors (SE) and Monte Carlo standard error (MC SE) of the 500 Monte Carlo replicates for the data generated from the Student-t with 4 degrees of freedom. The mean AIC and BIC are reported for the SLn and SLt models.

\begin{tabular}{cccccccc}
\hline \multirow{2}{*}{ Sample Size } & & \multicolumn{3}{c}{ SLn } & & SLt \\
\cline { 3 - 8 } & TRUE & EM & SE & MC SE & EM & SE & MC SE \\
\hline \multirow{2}{*}{$\beta_{0}=1.000$} & 0.964 & 0.260 & 0.176 & 1.011 & 0.146 & 0.131 \\
& $\beta_{1}=0.500$ & 0.515 & 0.189 & 0.193 & 0.498 & 0.152 & 0.152 \\
& $\gamma_{0}=0.741$ & 0.689 & 0.092 & 0.094 & 0.697 & 0.094 & 0.096 \\
& $\gamma_{1}=0.300$ & 0.316 & 0.157 & 0.157 & 0.320 & 0.159 & 0.160 \\
$\gamma_{2}=-0.500$ & -0.410 & 0.104 & 0.087 & -0.511 & 0.115 & 0.109 \\
& $\sigma^{2}=1.000$ & 1.406 & 0.219 & 0.079 & 1.024 & 0.113 & 0.089 \\
& $\rho=0.600$ & 0.596 & 0.379 & 0.161 & 0.573 & 0.234 & 0.191 \\
$\nu=4$ & & & & 6.904 & & \\
AIC & & 846.417 & & & 824.338 & \\
BIC & & 849.938 & & & 827.859 & \\
\hline \multirow{5}{*}{500} & $\beta_{0}=1.000$ & 0.916 & 0.162 & 0.115 & 1.001 & 0.092 & 0.091 \\
& $\beta_{1}=0.500$ & 0.536 & 0.126 & 0.130 & 0.506 & 0.101 & 0.104 \\
& $\gamma_{0}=0.741$ & 0.643 & 0.066 & 0.065 & 0.747 & 0.081 & 0.078 \\
$\gamma_{1}=0.300$ & 0.255 & 0.105 & 0.108 & 0.307 & 0.127 & 0.128 \\
& $\gamma_{2}=-0.500$ & -0.395 & 0.074 & 0.060 & -0.503 & 0.079 & 0.077 \\
& $\sigma^{2}=1.000$ & 1.414 & 0.173 & 0.051 & 1.012 & 0.076 & 0.063 \\
& $\rho=0.600$ & 0.678 & 0.240 & 0.089 & 0.596 & 0.147 & 0.133 \\
& $\nu=4$ & & & & 4.442 & & \\
AIC & & 1704.538 & & & 1658.626 & \\
BIC & & 849.938 & & & 827.859 & \\
\hline$\beta_{0}=1.000$ & 0.894 & 0.104 & 0.076 & 1.005 & 0.061 & 0.063 \\
$\beta_{1}=0.500$ & 0.533 & 0.089 & 0.091 & 0.500 & 0.076 & 0.074 \\
$\gamma_{0}=0.741$ & 0.642 & 0.045 & 0.045 & 0.746 & 0.057 & 0.055 \\
$\gamma_{1}=0.300$ & 0.248 & 0.075 & 0.075 & 0.303 & 0.090 & 0.089 \\
$\gamma_{2}=-0.500$ & -0.389 & 0.051 & 0.043 & -0.502 & 0.055 & 0.056 \\
$\sigma^{2}=1.000$ & 1.421 & 0.116 & 0.034 & 1.004 & 0.053 & 0.044 \\
$\rho=0.600$ & 0.723 & 0.142 & 0.047 & 0.596 & 0.098 & 0.095 \\
$\nu=4$ & & & & 4.166 & & \\
AIC & & 3427.196 & & & 3331.052 & \\
BIC & & 3432.103 & & & 3335.960 & \\
\hline 1000 & & & & & &
\end{tabular}

Thus, for this study, data are generated from the normal, Student's-t, and slash distributions, respectively, with increasing sample sizes, say, $n=250,500$, and 1000. The components of $\mathbf{w}_{i}^{\top}=\left(1, w_{i 1}, w_{i 2}\right)$ are generated from a Uniform $(-1,1)$ and Normal $(0,1)$, respectively. To consider the exclusion restriction we let $\mathbf{x}_{i}^{\top}=\left(1, w_{i 1}\right), i \in\{1, \ldots, n\}$. For all scenarios $\gamma=\left(\beta_{0 d}, 0.3,-0.5\right)$ and $\beta=(1,0.5)$. The $\beta_{0 d}$ is selected as the $-25 \%$ quantile times $\sigma^{2}$ of the generating distribution in order to guarantee an average missing rate of $25 \%$. Finally, the scale parameters are set at $\sigma^{2}=1$ and $\rho=0.6$.

From Table 1 we can see that both SLn e SLt models recover closely the original values of the parameters for all sample sizes, but being more precise as the sample size $n$ increases. Also, it is possible to see that the estimated standard errors for the parameters are very close to the Monte Carlo standard deviations. The model selection criterion does not differentiate the fit, which is expected since the SLn model is a especial case of the SLt model when $\nu=+\infty$ and $\nu$ is estimated in high values for the SLt model.

Now, under the same specification of the parameters values, we generate data from a Student's-t with $\nu=4$. The results are given in Table 2 From this table we can see that the SLn model does not perform well when data is generated from a Student's-t distribution with $\nu=4$. While the SLt fit seems to be adequate and improves as the sample sizes increase, the SLn model present 
Table 3: Simulation study 1. Mean estimates (EM), mean standard errors (SE) and Monte Carlo standard error (MC $\mathrm{SE})$ of the 500 Monte Carlo replicates for the data generated from the Slash with 1.43 degrees of freedom. The mean AIC and BIC are reported for the SLn and SLt models.

\begin{tabular}{|c|c|c|c|c|c|c|c|}
\hline \multirow[t]{2}{*}{ Sample Size } & \multirow[b]{2}{*}{ TRUE } & \multicolumn{3}{|c|}{ SLn } & \multicolumn{3}{|c|}{ SLt } \\
\hline & & EM & SE & MC SE & EM & SE & MC SE \\
\hline \multirow[t]{10}{*}{250} & $\beta_{0}=1.000$ & 0.989 & 0.392 & 0.235 & 1.020 & 0.197 & 0.167 \\
\hline & $\beta_{1}=0.500$ & 0.510 & 0.238 & 0.248 & 0.492 & 0.181 & 0.184 \\
\hline & $\gamma_{0}=0.925$ & 0.651 & 0.100 & 0.092 & 0.751 & 0.116 & 0.108 \\
\hline & $\gamma_{1}=0.300$ & 0.214 & 0.152 & 0.153 & 0.255 & 0.175 & 0.176 \\
\hline & $\gamma_{2}=-0.500$ & -0.327 & 0.099 & 0.083 & -0.412 & 0.107 & 0.103 \\
\hline & $\sigma^{2}=1.000$ & 1.781 & 0.395 & 0.091 & 1.272 & 0.145 & 0.113 \\
\hline & $\rho=0.600$ & 0.543 & 0.466 & 0.181 & 0.561 & 0.271 & 0.204 \\
\hline & $\nu=1.43$ & & & & 10.273 & & \\
\hline & AIC & \multicolumn{3}{|c|}{936.927} & \multicolumn{3}{|c|}{907.287} \\
\hline & BIC & & 940.448 & & & 910.808 & \\
\hline \multirow[t]{10}{*}{500} & $\beta_{0}=1.000$ & 0.904 & 0.292 & 0.163 & 1.016 & 0.126 & 0.116 \\
\hline & $\beta_{1}=0.500$ & 0.534 & 0.162 & 0.178 & 0.501 & 0.124 & 0.126 \\
\hline & $\gamma_{0}=0.925$ & 0.645 & 0.073 & 0.064 & 0.747 & 0.081 & 0.076 \\
\hline & $\gamma_{1}=0.300$ & 0.208 & 0.103 & 0.108 & 0.250 & 0.122 & 0.126 \\
\hline & $\gamma_{2}=-0.500$ & -0.315 & 0.068 & 0.058 & -0.408 & 0.073 & 0.074 \\
\hline & $\sigma^{2}=1.000$ & 1.807 & 0.490 & 0.057 & 1.246 & 0.093 & 0.080 \\
\hline & $\rho=0.600$ & 0.648 & 0.336 & 0.097 & 0.574 & 0.169 & 0.149 \\
\hline & $\nu=1.43$ & \multirow{2}{*}{\multicolumn{3}{|c|}{1888.094}} & 5.226 & & \\
\hline & AIC & & & & \multicolumn{3}{|c|}{1821.601} \\
\hline & BIC & & 1892.30 & & & 1825.815 & \\
\hline \multirow[t]{10}{*}{1000} & $\beta_{0}=1.000$ & 0.866 & 0.225 & 0.095 & 1.014 & 0.083 & 0.080 \\
\hline & $\beta_{1}=0.500$ & 0.532 & 0.120 & 0.119 & 0.495 & 0.092 & 0.089 \\
\hline & $\gamma_{0}=0.925$ & 0.643 & 0.054 & 0.045 & 0.750 & 0.057 & 0.054 \\
\hline & $\gamma_{1}=0.300$ & 0.203 & 0.080 & 0.075 & 0.250 & 0.093 & 0.088 \\
\hline & $\gamma_{2}=-0.500$ & -0.309 & 0.057 & 0.041 & -0.409 & 0.055 & 0.053 \\
\hline & $\sigma^{2}=1.000$ & 1.816 & 0.263 & 0.035 & 1.236 & 0.067 & 0.056 \\
\hline & $\rho=0.600$ & 0.701 & 0.256 & 0.046 & 0.583 & 0.107 & 0.104 \\
\hline & $\nu=1.43$ & & & & 4.415 & & \\
\hline & AIC & \multicolumn{3}{|c|}{3803.225} & \multicolumn{3}{|c|}{3661.727} \\
\hline & BIC & \multicolumn{3}{|c|}{3808.132} & \multicolumn{3}{|c|}{3666.635} \\
\hline
\end{tabular}

bias in the estimation of the parameters that increase as sample sizes increase. Although this may seem counter-intuitive it is not, with a bigger sample size more data is observed at the tail, due to the fat tail of the Studen-t distribution, and as expected affect the SLn model estimation. The only parameter that seems not to suffer under the SLn model is $\beta_{1}$. We can see that as $n$ increases and more observation in the tails are available, the estimation of $\nu$ improves drastically. Also we can see that the model selection criterion (AIC and BIC) are in favor of the SLt model, this is, also expected since the SLt is the true generating model and the SLn does not provide an adequate fit for the heavy-tailed data.

To study the robustness of the SLn and SLt model under model misspecification, data are now generated from the slash distribution with heavy tails (degrees of freedom 1.43) keeping the same specification of the other parameters values. The results are presented in Table 3 The model selection criteria are in favor of the SLt model, as expected, indicating that under model misspecification it provides a more apropriate fit.

As can be seen, the estimates of $\boldsymbol{\beta}$ is better than for $\boldsymbol{\gamma}$ for the SLn and SLt models. However, the bias for the SLn model is larger than the SLt model, especially, when the sample sizes increase. Moreover, the estimates for the SLt model are more stable for the different sample sizes. For example, it is clear that as sample sizes increase the bias estimation for $\rho$ under the SLn model increases drastically while the SLt model does not suffer significant variation. As sample size increase we can see that the estimate of $\nu$ reduces, indicating that is necessary a heavy-tailed model to provide an appropriate fit. Finally, the standard error estimates for the SLt model is stable under all scenarios. 

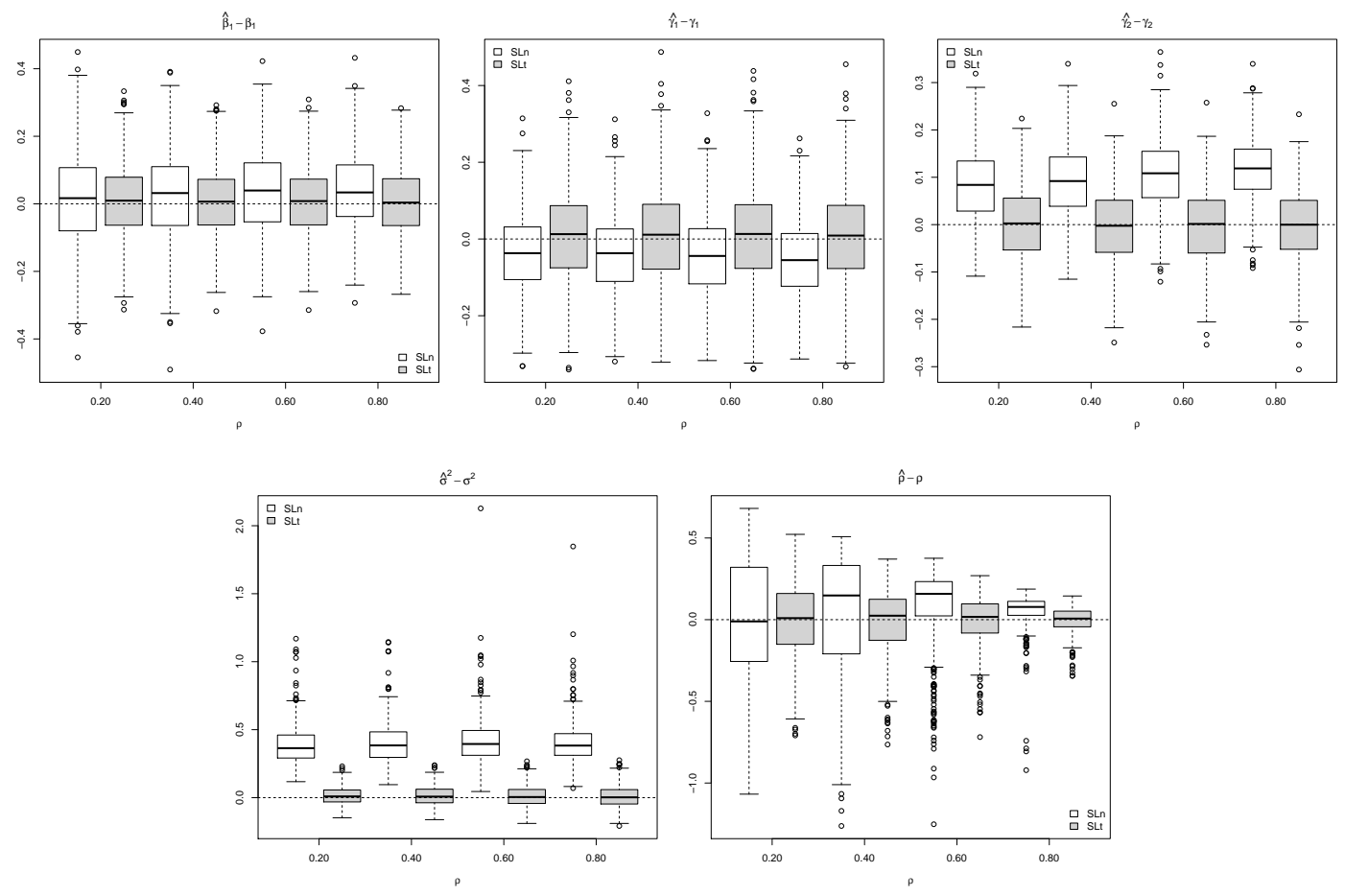

Figure 1: Show the boxplots of the estimated minus the true parameter for the SLn (white) and SLt (gray) for the different dependence values, $\rho=(0.20,0,40,0.60,0.80)$.

\subsection{Dependence variation}

In this section, we set the values of the parameters as in the previous section and generate data from the SLt model with $\nu=4$. To study the effect of the dependence parameter, we vary $\rho$ from mild, $\rho=0.20$, to strong, $\rho=0.80$, dependence by a step of 0.20 .

Figure 1 show the boxplots of the Monte Carlo fits of the SLn model (in white) and SLt model (in gray) for the 500 Monte Carlo replicates. From this figure we can see that the SLn model provides large bias estimates with a larger variance. However, the SLt model provides accurate estimates for all parameters, except for the dependence parameter $\rho$ where the variability reduces as the dependence gets bigger. Although the SLn has a larger bias, the reduction in the estimates variability observed for the SLt model is also observed for this model.

\subsection{Missing variation}

To understand the effects of missing values in the model we vary the average percentage of missing from $10 \%, 25 \%$, and $50 \%$. We kept the other configuration of the SLt generating model from the previous section. Figure 2 shows the boxplots of the centered estimates of the parameters. As previously observed the SLt model have very stable results while the SLn have lager bias for all parameters. An interesting observation is that the variance for $\gamma$ are larger for the SLt model with lower missing values than for largers ones.

Overall, it is clear that the proposed algorithms provide good point estimates as well as adequate standard errors estimation (Section 5.1 for both SLn and SLt models. Moreover, we verified that the SLt model is more robust than the SLn model for different variation on the characteristics of the generating model, as dependence (Section 5.2), missing (Section 5.3), and model misspecification (Section 5.1.

\section{Application}

We illustrate the proposed algorithms with the analysis of two real data sets. The analysis was performed using the $\mathrm{R}$ package HeckmanEM available on Github (https://github.com/marcosop/HeckmanEM). 

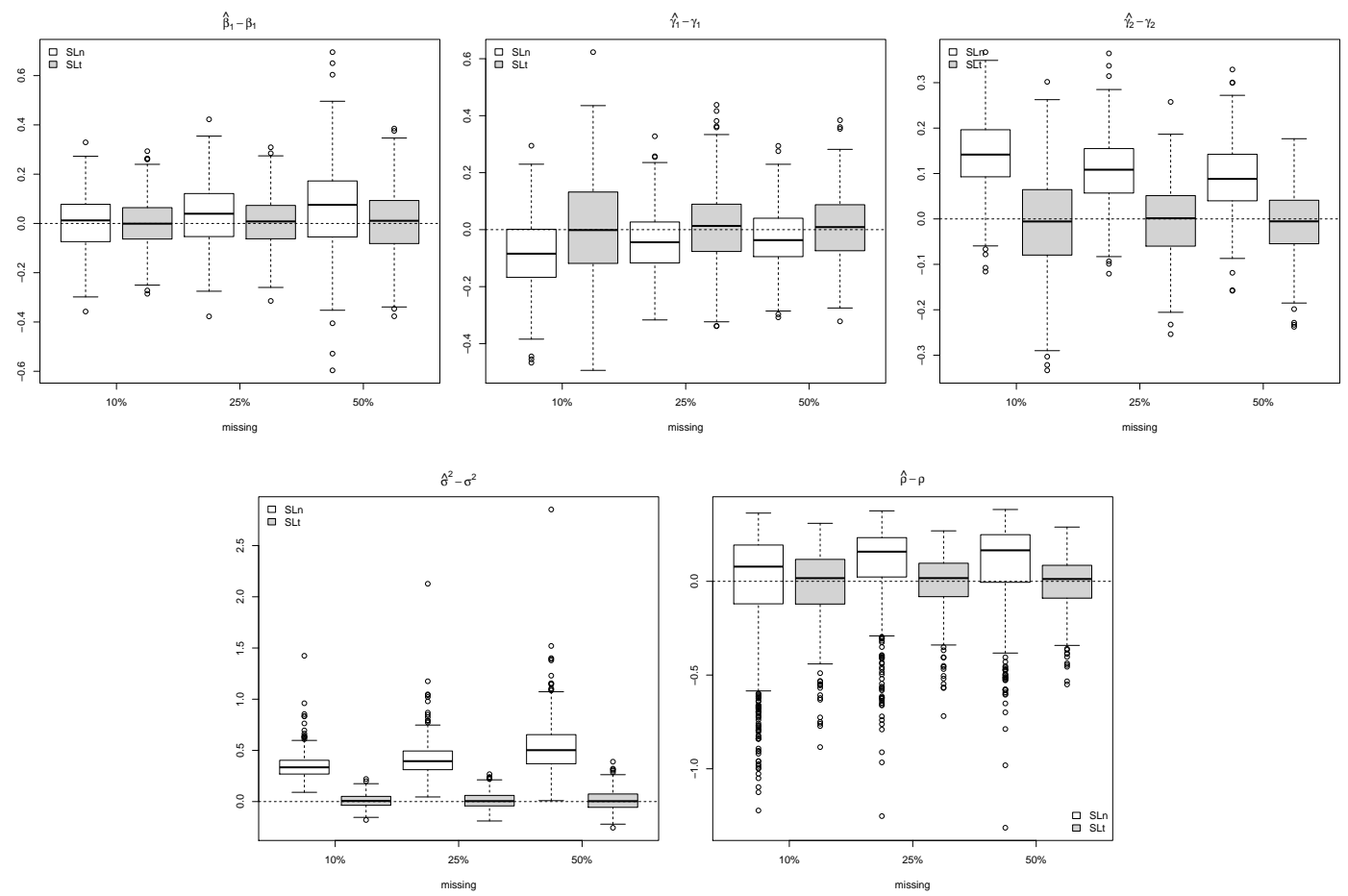

Figure 2: Show the boxplots of the estimated minus the true parameter for the SLn (white) and SLt (gray) for the different missing values, $10 \%, 25 \%$, and $50 \%$.

\subsection{Ambulatory expenditures}

The first application concerns a study of ambulatory expenditures. The data are taken from Cameron and Trivedi (2009), which were re-analyzed by Marchenko and Genton (2012) using ML estimation procedure in St at a. More recently, the data were also revisited by Ding (2014) using Bayesian procedures.

In our analysis, we choose the same set of covariates as Marchenko and Genton (2012), we choose the ln of ambulatory expenditures (ambexp) as the outcome variable. The covariates in the outcome equation are $\mathbf{x}=(1$, age, female, educ, blhisp, totchr, ins $)$, including age, gender, education status, ethnicity, number of chronic diseases and insurance status, respectively. The exclusion restriction assumption holds by including the income variable into the selection equation, i.e., $\mathbf{w}=(\mathbf{x}$, income $)$. The dataset contains 3328 observations and there are 526 missing values of ambexp. More details about the data can be found in Chap. 16 of Cameron and Trivedi (2009).

The estimation results for the SLn model and the SLt model are presented in Table 4 As expected, we find similar results to those presented by Marchenko and Genton (2012) and Ding (2014). As noted by these authors, under the SLn model, the 95\% confidence interval of $\rho$ contain zero $(-0.560 ; 0.300)$, which indicates weak evidence of the SL bias. However, under the SLt model, the 95\% confidence interval of $\rho$ does not contain zero $(-0.369 ;-0.275)$, which suggests the existence of SL effect.

In order to identify atypical observations and/or model misspecification, we use the martingale-type residuals, as discussed by Massuia et al. (2015) (see also, Garay et al. 2016) in the context of for censored models. We propose to work with the $r_{M T_{i}}$ residual, which is given by:

$$
r_{M T_{i}}=\operatorname{sign}\left(r_{M_{i}}\right) \sqrt{-2\left[r_{M_{i}}+C_{i} \ln \left(C_{i}-r_{M_{i}}\right)\right]}, \quad i=1, \ldots, n
$$

where $r_{M_{i}}=C_{i}+\log \left(S\left(y_{i} ; \widehat{\boldsymbol{\theta}}\right)\right)$ is the martingale residual, with $C_{i}=0,1$ as defined in 8 , $\operatorname{sign}\left(r_{M_{i}}\right)$ denoting the sign of $r_{M_{i}}$ and $S\left(y_{i}, \widehat{\boldsymbol{\theta}}\right)=P_{\widehat{\boldsymbol{\theta}}}\left(Y_{2 i} \leq 0\right)$ evaluated in the ML estimates under the SLn model or the SLt model. A more detailed account of the martingale residual can be found in Therneau et al. (1990).

The normal probability plot of the $r_{M_{i}}$ residuals with generated envelopes is presented in Figure 3 We observe that the SLt model fit the data better than the SLn model, since, in that case, there are fewer observations which lie outside the envelopes. Moreover, Table 4 (bottom) presents some model selection criteria together with the values of the log-likelihood. The AIC and BIC values indicate that the SLt model with heavy tails presents a better fit than the SLn model, due to the departure of the data from normality. 

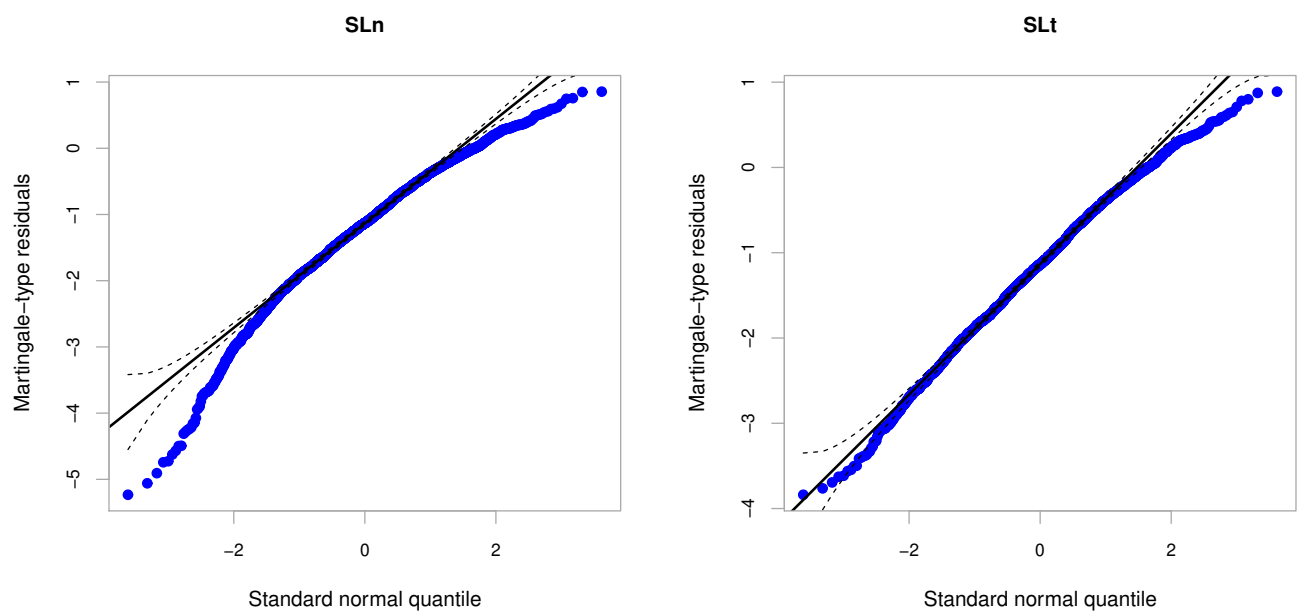

Figure 3: Ambulatory expenditures data. Envelopes of the martingale-type residuals $r_{M T_{i}}$ for the SLn and SLt models.

Table 4: Ambulatory expenditures Data. Parameter estimates and standard errors.

\begin{tabular}{lccccc}
\hline & \multicolumn{2}{c}{ SLn } & & \multicolumn{2}{c}{ SLt } \\
\cline { 6 - 6 } \cline { 5 - 6 } Parameter & EM & SE & & EM & SE \\
\hline Outcome model (ln expenditures) & & & & \\
\hline Intercept & 5.024 & 0.287 & & 5.192 & 0.222 \\
age & 0.213 & 0.024 & & 0.207 & 0.023 \\
female & 0.353 & 0.073 & & 0.310 & 0.060 \\
educ & 0.019 & 0.012 & & 0.018 & 0.010 \\
blhisp & -0.222 & 0.065 & & -0.195 & 0.059 \\
totchrn & 0.543 & 0.054 & & 0.514 & 0.042 \\
ins & -0.029 & 0.054 & -0.052 & 0.052 \\
\hline Selection model & & & & \\
\hline Intercept & -0.681 & 0.202 & -0.760 & 0.217 \\
age & 0.088 & 0.027 & & 0.099 & 0.030 \\
female & 0.666 & 0.061 & & 0.732 & 0.067 \\
educ & 0.062 & 0.013 & & 0.065 & 0.014 \\
blhisp & -0.365 & 0.063 & & -0.396 & 0.067 \\
totchrn & 0.814 & 0.069 & & 0.920 & 0.084 \\
ins & 0.171 & 0.065 & & 0.182 & 0.070 \\
income & 0.003 & 0.001 & & 0.003 & 0.001 \\
\hline$\sigma$ & 1.270 & 0.019 & & 1.195 & 0.023 \\
$\rho$ & -0.131 & 0.220 & & -0.321 & 0.140 \\
$\nu$ & & & 12.928 & \\
\hline AIC & 11674.44 & 11646.15 \\
BIC & 11680.55 & 11652.26 \\
\hline
\end{tabular}

\subsection{RAND Health Insurance data}

The second application concerns a study from RAND Health Insurance Experiment (RAND HIE), which is a comprehensive study of health care cost, utilization, and outcome in the United States. This data set is used by Cameron and Trivedi (2009) to analyse how the patients use of health services is affected by types of randomly assigned health insurance. More recently, the data were also revisited by Zhao et al. (2020) considering a SLn model. 
In our analysis, we choose the same set of variables as Zhao et al. (2020), we choose the ln of the medical expenses of individual $(m e d d o l)$ as the outcome variable. The covariates in the outcome equation are:

$\mathbf{x}=(1, \log c, i d p$, lpi, fmde, physlm, disea, hlthg, hlthf, hlthp, linc, lfam, educdec, xage, female, child, fchild, black $)$,

including the $\log$ of coinsurance rate plus $1(\log c=\ln ($ coins +1$))$, the dummy for individual deductible plan $(i d p)$, the $\log$ of participation incentive payment $(l p i)$, an artificial variable $f m d e$ that is 0 if $i d p=1$ and $\ln (\max (1, \operatorname{mde} /(0.01 *$ coins $)))$ otherwise (where mde is the maximum expenditure offer), physical limitations (physlm), the number of chronic diseases (disea), dummy variables for good ( $h l t h g)$, fair $(h l t h f)$, and poor $(h l t h p)$ self-rated health (where the baseline is excellent self-rated health), the log of family income (linc), the log of family size (lfam), education of household head in years (educdec), age of individual in years (xage), a dummy variable for female individuals (female), a dummy variable for individuals younger than 18 years (child), a dummy variable for female individuals younger than 18 years $(f c h i l d)$, and a dummy variable for black household heads (black). The selection variable is binexp which indicates whether the medical expenses are positive and without exclusion, we consider that $\mathbf{x}=\mathbf{w}$.

For our analysis, a subsample was selected so that study year is 2 and educdec is not NA. Out of 5574 observations, 1293 of meddol (medical expenses) are 0 which means the outcome variable $l n$ of meddol is unobserved, and 4281 of meddol are positive (means that the outcome variable $l n$ of meddol is available). The data is available in the R package sampleSelection. The results for the SLn model and the SLt model are presented in Table 5 as in the previous real analysis, the ML estimates for the SLn model are the same than those reported in Zhao et al. (2020) and also at http: / / cameron.econ.ucdavis.edu/ mmabook/mma16p3selection.txt. In this case clearly, the $95 \%$ confidence interval of $\rho$ does not contain zero, which suggests the existence of SL effect under both models (SLn and SLt).

The normal probability plot of the $r_{M_{i}}$ residuals with generated envelopes is presented in Figure 4 As in the previous example, we observe that the SLt model fit the data better than the SLn model, which is corroborated by the relative small value of the degrees of freedom $\nu$, which is $\hat{\nu}=8.809$. Moreover, the AIC and BIC values indicate that the SLt model with heavy tails presents a better fit than the SLn model, due to the departure of the data from normality.
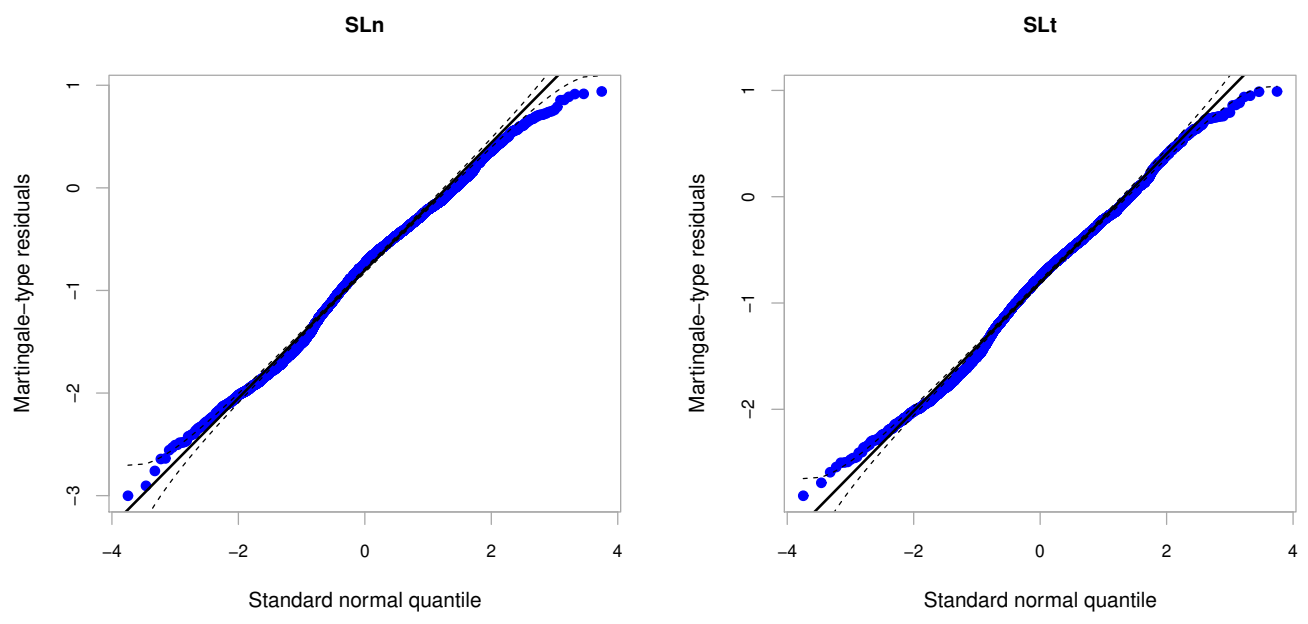

Figure 4: RAND HIE data. Envelopes of the martingale-type residuals $r_{M T_{i}}$ for the SLn and SLt models.

\section{Conclusions}

In this paper, a novel EM-type algorithm for the SLt model has been developed. In contrast with the existing literature, where to compute the ML estimates, available optimization procedures in standard programs, such as, the optim routine in R or the $\mathrm{ml}$ routine in stata, are used. As discussed by Zhao et al. (2020) (Sec. 6), a disadvantage of direct maximization of the log-likelihood function is that it may not converge unless good starting values are used. Our proposed EM-type algorithm for the SLt model uses closed-form expressions at the E-step, that rely on formulas of the mean and variance of a truncated Student's- $t$ distribution. The general formulas for these moments were derived efficiently by Galarza et al. (2020), for which we use the MomTrunc package in R. We also propose a slight modification to the EM-type algorithm proposed by Zhao et al. (2020), where the parameters in the M-step are updated by considering the outcome and sample selection as missing data. It is important to point out that the proposed EM algorithm perform more robustly than direct maximization, but is computationally costlier. The analysis of two real data sets provide strong evidence about the usefulness and effectiveness of our proposal. Moreover, intensive simulation studies show the vulnerability of the SLn model, as well as, the robustness of the SLt model. 
A PREPRINT - JUNE 16, 2020

Table 5: RAND HIE data. Parameter estimates and standard errors.

\begin{tabular}{|c|c|c|c|c|}
\hline \multirow[b]{2}{*}{ Parameter } & \multicolumn{2}{|c|}{ SLn } & \multicolumn{2}{|c|}{ SLt } \\
\hline & EM & SE & EM & SE \\
\hline \multicolumn{5}{|c|}{ Outcome model (ln of meddol) } \\
\hline Intercept & 2.155 & 0.253 & 2.358 & 0.242 \\
\hline $\log c$ & -0.073 & 0.033 & -0.067 & 0.032 \\
\hline$i d p$ & -0.146 & 0.063 & -0.152 & 0.061 \\
\hline lpi & 0.014 & 0.011 & 0.014 & 0.010 \\
\hline fmde & -0.024 & 0.019 & -0.028 & 0.018 \\
\hline physlm & 0.350 & 0.073 & 0.339 & 0.070 \\
\hline disea & 0.028 & 0.004 & 0.028 & 0.0036 \\
\hline hlthg & 0.156 & 0.052 & 0.145 & 0.050 \\
\hline hlthf & 0.442 & 0.092 & 0.462 & 0.089 \\
\hline hlthp & 0.989 & 0.167 & 0.881 & 0.165 \\
\hline linc & 0.120 & 0.024 & 0.110 & 0.023 \\
\hline lfam & -0.157 & 0.048 & -0.180 & 0.047 \\
\hline educdec & 0.017 & 0.009 & 0.016 & 0.009 \\
\hline xage & 0.006 & 0.002 & 0.005 & 0.002 \\
\hline female & 0.540 & 0.061 & 0.503 & 0.060 \\
\hline child & -0.202 & 0.098 & -0.192 & 0.093 \\
\hline fchild & -0.554 & 0.100 & -0.526 & 0.095 \\
\hline black & -0.518 & 0.070 & -0.502 & 0.072 \\
\hline \multicolumn{5}{|c|}{ Selection model } \\
\hline Intercept & -0.220 & 0.191 & -0.228 & 0.207 \\
\hline $\log c$ & -0.108 & 0.025 & -0.129 & 0.028 \\
\hline$i d p$ & -0.110 & 0.048 & -0.105 & 0.053 \\
\hline lpi & 0.030 & 0.009 & 0.033 & 0.010 \\
\hline fmde & 0.002 & 0.016 & 0.005 & 0.018 \\
\hline physlm & 0.285 & 0.073 & 0.335 & 0.084 \\
\hline disea & 0.021 & 0.004 & 0.024 & 0.004 \\
\hline hlthg & 0.056 & 0.043 & 0.055 & 0.047 \\
\hline hlthf & 0.223 & 0.082 & 0.2467 & 0.091 \\
\hline hlthp & 0.796 & 0.187 & 0.904 & 0.230 \\
\hline linc & 0.055 & 0.017 & 0.054 & 0.018 \\
\hline lfam & -0.032 & 0.040 & -0.041 & 0.044 \\
\hline educdec & 0.032 & 0.008 & 0.037 & 0.008 \\
\hline xage & -0.001 & 0.002 & -0.001 & 0.002 \\
\hline female & 0.413 & 0.053 & 0.463 & 0.059 \\
\hline child & 0.059 & 0.079 & 0.082 & 0.087 \\
\hline fchild & -0.401 & 0.079 & -0.456 & 0.087 \\
\hline black & -0.587 & 0.051 & -0.646 & 0.055 \\
\hline$\sigma$ & 1.570 & 0.027 & 1.374 & 0.027 \\
\hline$\rho$ & 0.736 & 0.037 & 0.667 & 0.047 \\
\hline$\nu$ & & & 8.809 & \\
\hline AIC & \multicolumn{2}{|c|}{20342.22} & \multicolumn{2}{|c|}{20284.12} \\
\hline BIC & \multicolumn{2}{|c|}{20348.85} & \multicolumn{2}{|c|}{20290.75} \\
\hline
\end{tabular}

A promising avenue for future research is to consider a generalization of the SLt model to the scale mixtures of skew-normal (SMSN) distribution (Lachos et al. 2010), this rich family of SMSN distributions include some well-known multivariate asymmetric heavy-tailed and symmetric distributions, such as, the skew-t (Azzalini and Capitanio 1999) and the family of scale-mixture of normal distributions (Lange and Sinsheimer 1993). Another possible extension, includes likelihood-based treatment for the multivariate SL model (Tauchmann, 2010). 


\section{Acknowledgments}

This paper was written while Marcos O. Prates was a visiting professor in the Department of Statistics at the University of Connecticut (UConn). In addition to the support of UConn, the professor would like also to thank Conselho Nacional de Desenvolvimento Cientfico e Tecnolgico (CNPq) for partial financial support.

\section{References}

Ahn, H. and J. L. Powell (1993). Semiparametric estimation of censored selection models with a nonparametric selection mechanism. Journal of Econometrics 58(1-2), 3-29.

Arellano-Valle, R. and H. Bolfarine (1995). On some characterizations of the t-distribution. Statistics \& Probability Letters 25 , 79-85.

Azzalini, A. and A. Capitanio (1999). Statistical applications of the multivariate skew-normal distribution. Journal of the Royal Statistical Society, Series B 61, 579-602.

Basford, K., D. Greenway, G. McLachlan, and D. Peel (1997). Standard errors of fitted component means of normal mixtures. Computational Statistics 12, 1-18.

Cameron, A. C. and P. K. Trivedi (2009). Microeconometrics using Stata, Volume 5. Stata press College Station, TX.

Das, M., W. K. Newey, and F. Vella (2003). Nonparametric estimation of sample selection models. The Review of Economic Studies 70(1), 33-58.

Ding, P. (2014). Bayesian robust inference of sample selection using selection-t models. Journal of Multivariate Analysis 124, 451-464.

Galarza, C. E., R. Kan, and V. H. Lachos (2020). MomTrunc: Moments of Folded and Doubly Truncated Multivariate Distributions. $R$ Package Version 5.87 URL http://CRAN.R-project.org/package=MomTrunc.

Garay, A. M., V. H. Lachos, and T.-I. Lin (2016). Nonlinear censored regression models with heavy-tailed distributions. Statistics and its Interface 9(3), 281-293.

Heckman, J. (1974). Shadow prices, market wages, and labor supply. Econometrica: Journal of the Econometric Society, 679-694.

Heckman, J. (1979). Sample selection bias as a specification error. Econometrica 47(1), 153-161.

Henningsen, A., O. Toomet, and S. Petersen (2019). sampleSelection: Sample selection models. R Package Version 1.2-0 URL https://cran.r-project.org/web/packages/sampleSelection/index.html.

Kai, L. (1998). Bayesian inference in a simultaneous equation model with limited dependent variables. Journal of Econometrics 85(2), 387-400.

Kim, H.-J., T. Roh, and T. Choi (2019). Bayesian analysis of semiparametric Bernstein polynomial regression models for data with sample selection. Statistics 53(5), 1082-1111.

Lachos, V. H., P. Ghosh, and R. B. Arellano-Valle (2010). Likelihood based inference for skew-normal independent linear mixed models. Statistica Sinica 20, 303-322.

Lange, K. L. and J. S. Sinsheimer (1993). Normal/independent distributions and their applications in robust regression. J. Comput. Graph. Stat 2, 175-198.

Lee, L.-F. (1983). Generalized econometric models with selectivity. Econometrica: Journal of the Econometric Society, $507-512$.

Louis, T. A. (1982). Finding the observed information matrix when using the EM algorithm. Journal of the Royal Statistical Society, Series B 44, 226-233.

Marchenko, Y. V. and M. G. Genton (2012). A heckman selection-t model. Journal of the American Statistical Association 107(497), 304-317.

Massuia, M. B., C. R. B. Cabral, L. A. Matos, and V. H. Lachos (2015). Influence diagnostics for Student-t censored linear regression models. Statistics 49(5), 1074-1094.

Matos, L. A., V. H. Lachos, N. Balakrishnan, and F. V. Labra (2013). Influence diagnostics in linear and nonlinear mixed-effects models with censored data. Computational Statistics \& Data Analysis 57.

Matos, L. A., M. O. Prates, M. H. Chen, and V. H. Lachos (2013). Likelihood-based inference for mixed-effects models with censored response using the multivariate-t distribution. Statistica Sinica 23, 1323-1342.

Meilijson, I. (1989). A fast improvement to the EM algorithm on its own terms. Journal of the Royal Statistical Society, Series B (Methodological) 51(1), 127-138.

Tauchmann, H. (2010). Consistency of Heckman-type two-step estimators for the multivariate sample-selection model. Applied Economics 42(30), 3895-3902.

Therneau, T. M., P. M. Grambsch, and T. R. Fleming (1990). Martingale-based residuals for survival models. Biometrika 77(1), $147-160$.

Vaida, F. and L. Liu (2009). Fast implementation for normal mixed effects models with censored response. Journal of Computational and Graphical Statistics 18(4), 797-817. 
A PREPRINT - JUNE 16, 2020

Wooldridge, J. M. (2010). Econometric analysis of cross section and panel data. MIT press.

Zhao, J., H.-J. Kim, and H.-M. Kim (2020). New EM-type algorithms for the Heckman selection model. Computational Statistics \& Data Analysis 146, 106930. 